\title{
An algebraic approach to the scattering equations
}

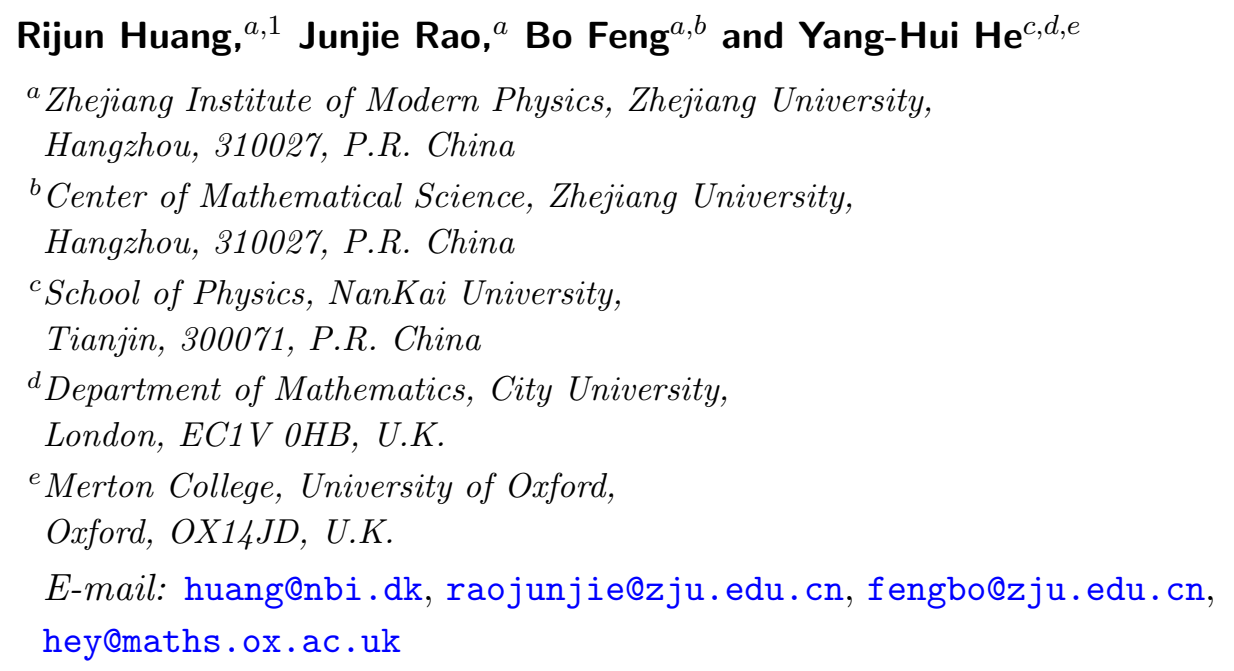

ABSTRACT: We employ the so-called companion matrix method from computational algebraic geometry, tailored for zero-dimensional ideals, to study the scattering equations. The method renders the CHY-integrand of scattering amplitudes computable using simple linear algebra and is amenable to an algorithmic approach. Certain identities in the amplitudes as well as rationality of the final integrand become immediate in this formalism.

Keywords: Scattering Amplitudes, Differential and Algebraic Geometry, Gauge Symmetry

ArXiv EPRINT: 1509.04483

\footnotetext{
${ }^{1}$ The unusual ordering of authors is just to let authors get proper recognition of contributions under outdated practice in China.
} 


\section{Contents}

1 Introduction 1

2 Review of tree-level scattering equations 3

3 The mathematical framework $\quad 6$

3.1 Warmup 7

4 Illustrative examples $\quad 8$

4.1 Four-point amplitudes $\quad 9$

4.1.1 Scalar $\phi^{3}$ theory 9

$\begin{array}{lll}\text { 4.1.2 Yang-Mills theory } & 10\end{array}$

$\begin{array}{lll}\text { 4.1.3 Gravity } & 11\end{array}$

$\begin{array}{lll}4.2 & \text { Five-point amplitudes } & 12\end{array}$

$\begin{array}{lll}\text { 4.2.1 Scalar } \phi^{3} \text { theory } & 13\end{array}$

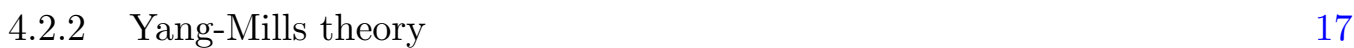

$\begin{array}{ll}\text { 4.2.3 Gravity and } n \text {-point KLT relations } & 19\end{array}$

$\begin{array}{lll}4.3 & \text { Six-point amplitudes } & 20\end{array}$

$\begin{array}{lll}\text { 4.3.1 Scalar } \phi^{3} \text { theory } & 21\end{array}$

4.3.2 Yang-Mills theory 23

$\begin{array}{ll}4.4 & \text { Seven-point amplitudes }\end{array}$

5 Conclusions and outlook $\quad 29$

\section{Introduction}

In last couple of years, amazing progress has been made by Cachazo, He and Yuan [CHY] in a series of papers [1-5], where tree-level amplitudes of a host of quantum field theories can be calculated using solutions of a set of algebraic equations. These are called the scattering equations and appear in the literature in a variety of contexts [6-14].

The mysterious relationship between the CHY approach and the standard QFT paradigm has been explained from different points of view. In [15], using the BCFW on-shell recursion relation $[16,17]$ the validity of the $\mathrm{CHY}$ construction for $\phi^{3}$ theory and Yang-Mills theories has been proven. A broader understanding is achieved using ambitwistor string theory [18-28], where using different world-sheet fields, different integrands in the CHY approach for different theories - which we will call CHY-integrands, a function of the coordinates $z_{i}$ in a Riemann surface - have been derived alongside with the natural appearance of scattering equations. A nice point of ambitwistor approach is that it provides the natural framework for loop scattering equations as studied in [21, 24], which 
lead to a breakthrough in [28]. A third understanding is given in [29], where inspired by the field theory limit of string theory, a dual model has been introduced, based on which a direct connection between the CHY approach and the standard Feynman diagram method has been established not only at the tree-level in [30,31], but also at the one-loop level (at least for $\phi^{3}$ theory) in [32] (see also [33]). ${ }^{1}$

Although conceptually the $\mathrm{CHY}$ approach is remarkable and very useful for many theoretical studies of properties of scattering amplitudes, when applying to real evaluation, one faces the problem of solving scattering equations, which has $(n-3)$ ! solutions in general. Furthermore, when $n \geq 6$, one encounters polynomials of degree exceeding five, rendering analytic solutions in radicals hopeless. Nevertheless, while the solutions can be very complicated, when putting them back into the CHY integrand and summing up, one obtains simple rational functions. These observations have led people to wonder if there is a better way to evaluate the CHY-integrand without explicitly solving the scattering equations. In [35], using classical formulas of Vieta, which relate the sums of roots of polynomials to the coefficients of these polynomials, analytic expression can be obtained without solving roots explicitly. More general algorithms are given by two works. In one approach [36], using known results for scalar $\phi^{3}$ theory, one can iteratively decompose the 4-regular graph determined by the corresponding CHY-integrand to building blocks related to $\phi^{3}$ theory, thus finishing the evaluation. In another approach [30,31], by careful analysis of pole structures, the authors wrote down a mapping rule, so that from the related CHY-integrand, one can read out contributions of corresponding Feynman diagrams.

Both approaches are powerful and have avoided the need of solving the scattering equations explicitly. Furthermore, based on these perspectives, especially the mapping rule, one can use Feynman diagrams to construct the CHY-integrand. These results produce a very interesting phenomenon: two different $C H Y$-integrands can produce the same result. For example, there are two very different CHY-integrands for scalar $\phi^{4}$ theory: one is given in [5], while another one is given in $[30,31]$. We are naturally led to wonder how to explain the equivalence of different CHY-integrands.

In fact, as a rational function of coordinates $z_{i}$ on a Riemann surface, the equivalence can occur on three different levels.

1. At the first level, their equivalence is pure algebraic, i.e., through some algebraic manipulations, one rational expression can be transformed to another one. For example, for 4-point amplitudes of $\phi^{3}$ theory, on the one hand we have the integrand $I_{1}=$ $\frac{1}{z_{12} z_{23} z_{34} z_{41}} \frac{1}{z_{12} z_{23} z_{34} z_{41}}$ where we have defined $z_{i j}=z_{i}-z_{j}$ which gives a contribution of $\frac{1}{s_{12}}+\frac{1}{s_{41}}$. On the other hand, we have the integrands $I_{21}=\frac{1}{z_{12} z_{23} z_{34} z_{41}} \frac{1}{z_{12} z_{24} z_{43} z_{31}}$ which gives $-\frac{1}{s_{12}}$ and the integrand $I_{22}=\frac{1}{z_{12} z_{23} z_{34} z_{41}} \frac{1}{z_{13} z_{32} z_{24} z_{41}}$ which gives $-\frac{1}{s_{41}}$. It is easy to check algebraically that $I_{1}=-I_{21}-I_{22}$. Equivalences at this level is of course rather trivial and in order to proceed to the other two levels of equivalences, we need to change our viewpoint to algebraic geometry, i.e., to transform the scattering equations to a set of polynomials of $(n-3)$ variables, defining an ideals $\mathcal{I}$;

\footnotetext{
${ }^{1}$ Recently, inspired by the development of CHY-approach, a new method to construct all loop integrands for general massless quantum field theories has been proposed in [34].
} 
2. The difference of two CHY-integrands can be written as $J\left(I_{1}-I_{2}\right)=\frac{P}{Q}$ where both $P, Q$ are polynomials and $J$ is the Jacobian we will review shortly. If $P$ belongs to the ideal $\mathcal{I}$, then for each solution of the scattering equations $P=0$, thus at the second level we say that $I_{1}$ is equivalent to $I_{2}$;

3. However, in practice, most of the time something more complicated happens and we find that though $P$ does not belong to the ideal $\mathcal{I}$ and $J\left(I_{1}-I_{2}\right)=0$ when and only when we sum over all solutions. If this happens, we say that $I_{1}$ is equivalent to $I_{2}$ at the third level. It is clear that this is the most involved situation, and indeed, in practice this is the most frequently encountered.

Motivated by the above considerations and bearing in mind that indeed the most conducive perspective on studying the scattering equations is through the language of algebraic varieties and polynomial ideals [37, 38], we turn to this method of attack. The above problem thus translates to finding the sum over the rational function $\frac{P}{Q}$ evaluated at the roots of a zero-dimensional ideal $I$, and testing whether the sum is zero. Luckily, there is a theorem in commutative algebra, due to Stickelberger, which addresses the situation [39]. We will discuss the theorem and the associated algorithm in illustrative detail. It turns out that this method not only checks the equivalence at the third level, but also evaluates the integration without solving the scattering equations. In this sense, it is in the spirit of the methods in [36] and [30,31]. Although it is sometimes less efficient compared to these two methods, it does provide a very different angle to approach the problem and could have very advantageous repercussions.

The structure of the paper is as follows. We begin with a brief review of the treelevel scattering equations in section 2, before laying down the foundations of the theory of zero-dimensional ideals in section 3, especially that of companion matrices. We then illustrate the technique with ample computational examples in section 4 , before concluding with remarks in section 5 .

\section{Review of tree-level scattering equations}

In this section, we offer a brief review of tree-level scattering equations and the reader is referred to $[1-3,5]$ for details. The scattering equations are given by

$$
\mathcal{E}_{a} \equiv \sum_{b \neq a} \frac{s_{a b}}{z_{a}-z_{b}}=0, \quad a=1,2, \ldots, n
$$

where $s_{a b}=\left(k_{a}+k_{b}\right)^{2}=2 k_{a} \cdot k_{b}$, and $k_{a}$ with $a=1,2, \ldots, n$ are $n$ massless momenta for $n$-external particles and $z_{i}$ are complex variables living on $\mathbb{C P}^{1}$ with $n$ punctures. Although there are $n$ equations, only $(n-3)$ of them are linear independent after using the momentum conservation and massless conditions which translate to the following three relations

$$
\sum_{a} \mathcal{E}_{a}=0, \quad \sum_{a} \mathcal{E}_{a} z_{a}=0, \quad \sum_{a} \mathcal{E}_{a} z_{a}^{2}=0
$$

which are, in fact, the consequence of the $\mathrm{SL}(2, \mathbb{C})$ symmetry on the $\mathbb{C P}^{1}$. Because of this, we can insert only $(n-3)$ delta-function. To make sure the result does not depend on 
which three equations have been removed, we make following combination and define the measure $^{2}$

$$
\Omega(\mathcal{E}) \equiv z_{i j} z_{j k} z_{k i} \prod_{a \neq i, j, k} \delta\left(\mathcal{E}_{a}\right)
$$

with $z_{i j}=z_{i}-z_{j}$. With the above, the general tree-level amplitude is given by

$$
\mathcal{A}_{n}=\int \frac{\prod_{i=1}^{n} d z_{i}}{\operatorname{vol}(\operatorname{SL}(2, \mathbb{C}))} \Omega(\mathcal{E}) \mathcal{F}(z)=\int \frac{\prod_{i=1}^{n} d z_{i}}{d \omega} \Omega(\mathcal{E}) \mathcal{F}(z),
$$

where $d \omega=\frac{d z_{r} d z_{s} d z_{t}}{z_{r s} z_{s t} z_{t r}}$ comes after we use the Möbius SL(2, C) symmetry to fix the location of three of the variables $z_{r}, z_{s}, z_{t}$ by the Faddeev-Popov method. Different QFTs give different forms of the $C H Y$-integrand $\mathcal{F}(z)$. Invariance under the Möbius transformation requires $\mathcal{F}(z)$ to have proper transformation behaviors, i.e., under $z^{\prime}=\frac{a z+b}{c z+d}$, we have

$$
\mathcal{F}(z) \rightarrow\left(\prod_{i=1}^{n} \frac{\left(c z_{i}+d\right)^{4}}{(a d-b c)^{2}}\right) \mathcal{F}(z)
$$

To simplify expression (2.4) further, we integrate out the delta-functions to arrive at the key expression

$$
\mathcal{A}_{n}=\sum_{s o l} \frac{z_{i j} z_{j k} z_{k i} z_{r s} z_{s t} z_{t r}}{(-)^{i+j+k+r+s+t}|\Phi|_{i j k}^{r s t}} \mathcal{F}
$$

where three arbitrary indices $i, j, k$ correspond to three removed scattering equations while three arbitrary indices $r, s, t$ correspond to the above mentioned three fixed locations. The sum is over the solution set of the scattering equations, which is generically a discrete set of points. Furthermore, in the above, the Jacobian matrix $\Phi$ is calculated as ( $a$ for rows and $b$ for column)

$$
\Phi_{a b}=\frac{\partial \mathcal{E}_{a}}{\partial z_{b}}= \begin{cases}\frac{s_{a b}}{z_{a b}^{2}} & a \neq b \\ -\sum_{c \neq a} \frac{s_{a c}}{z_{a c}^{2}} & a=b\end{cases}
$$

and $|\Phi|_{i j k}^{r s t}$ is the determinant of $\Phi$ after removing the $i$-th, $j$-th and $k$-th rows and $r$-th, $s$-th and $t$-th columns.

Specific examples. Now we list some examples in the literatures $[2,3]$ (more can be found in [5]). According to the CHY formula, the integrand unifying scalars $(b=0)$, gluons $(b=1)$ and gravitons $(b=2)$ is given by

$$
\mathcal{F}_{b, n}=\left(\sum_{\alpha \in S_{n} / \mathbb{Z}_{n}} \frac{\operatorname{Tr}\left(T^{\alpha(1)} \ldots T^{\alpha(n)}\right)}{z_{\alpha(1) \alpha(2)} \ldots z_{\alpha(n) \alpha(1)}}\right)^{2-b}\left(\operatorname{Pf}^{\prime} \Psi\right)^{b},
$$

\footnotetext{
${ }^{2} \mathrm{~A}$ nice explanation of this fact can be found in $[15,37]$.
} 
where the sum is over permutations on $n$ elements by the symmetric group $S_{n}$, up to cyclic ordering of $\mathbb{Z}_{n}, \Psi$ is a $2 n \times 2 n$ antisymmetric matrix defined by $\Psi=\left(\begin{array}{cc}A-C^{t} \\ C & B\end{array}\right)$ (where $t$ is the transpose of the matrix), with $A, B, C$ being $n \times n$ matrices with components

$$
A_{a b}=\left\{\begin{array}{c}
\frac{k_{a} \cdot k_{b}}{z_{a}-z_{b}} \\
0
\end{array}, \quad B_{a b}=\left\{\begin{array}{l}
\frac{\epsilon_{a} \cdot \epsilon_{b}}{z_{a}-z_{b}} \\
0
\end{array}, \quad C_{a b}=\left\{\begin{array}{cc}
\frac{\epsilon_{a} \cdot k_{b}}{z_{a}-z_{b}} \\
-\sum_{c \neq a} \frac{\epsilon_{a} \cdot k_{c}}{z_{a}-z_{c}}
\end{array} \quad \text { for } \begin{array}{l}
a \neq b \\
a=b
\end{array},\right.\right.\right.
$$

and $\mathrm{Pf}^{\prime} \Psi$ is the reduced Pfaffian (square-root of the determinant) of $\Psi$ defined by

$$
\operatorname{Pf}^{\prime} \Psi=2 \frac{(-1)^{i+j}}{z_{i}-z_{j}} \operatorname{Pf} \Psi_{i j}^{i j},
$$

where $1 \leq i, j \leq n$ and $\Psi_{i j}^{i j}$ is the matrix $\Psi$ removing rows $i, j$ and columns $i, j$. We recall that the Pfaffian of a $2 n \times 2 n$ antisymmetric matrix can be computed as

$$
\operatorname{Pf} \Psi=\frac{1}{2^{n} n !} \sum_{\sigma \in S_{2 n}} \operatorname{sgn}(\sigma) \prod_{i=1}^{n} \Psi_{\sigma_{2 i-1} \sigma_{2 i}}
$$

where $\operatorname{sgn}(\sigma)$ is the signature of $\sigma \in S_{2 n}$. Importantly, $\operatorname{Pf} \Psi_{i j}^{i j}$ is non-zero on the solutions of scattering equations, while $\mathrm{Pf}^{\prime} \Psi$ is independent of the choice of $i, j$.

Specifically, we have that

- For color-ordered bi-adjoint scalar $\phi^{3}$ theory,

$$
\mathcal{F}_{\phi^{3}}=\frac{1}{z_{12}^{2} z_{23}^{2} \cdots z_{n 1}^{2}} .
$$

- For color-ordered Yang-Mills theory with ordering $\{1,2, \ldots, n\}$,

$$
\mathcal{F}_{\mathrm{YM}}=\frac{1}{z_{12} z_{23} \cdots z_{n 1}} \operatorname{Pf}^{\prime} \Psi
$$

- For gravity,

$$
\mathcal{F}_{\mathrm{G}}=\left(\mathrm{Pf}^{\prime} \Psi\right)^{2}=\frac{4}{\left(z_{i}-z_{j}\right)^{2}} \operatorname{Det}\left(\Psi_{i j}^{i j}\right)
$$

Having presented the above examples, let us go back to (2.6). As is clear from the expression, the right hand side is a rational function in the complex variables $z_{i}$. To employ methods developed in algebraic geometry, we need to associate solutions to a zerodimensional algebraic variety defined by some polynomials. In other words, we should rewrite $\mathcal{E}_{a}$ defined in (2.1) to an equivalent polynomial system. This has been done in a beautiful paper [37], where it has been shown that scattering equations are equivalent to following set of polynomials

$$
0=h_{m} \equiv \sum_{S \in A,|S|=m} k_{S}^{2} z_{S}, \quad 2 \leq m \leq n-2,
$$


where the sum is over all $\frac{n !}{(n-m) ! m !}$ subsets $S$ of $A=\{1,2, \ldots, n\}$ with exactly $m$ elements and $k_{S}=\sum_{b \in S} k_{b}$ and $z_{S}=\prod_{b \in S} z_{b}$. The algebraic geometry, notably the affine CalabiYau properties of (2.15), has been investigated in [38].

A very useful observation made in $[15,37]$ is that If all $k_{S}^{2} \neq 0$, then values of $z_{a}$ are all distinct. The set (2.15) has not fixed gauge. One of the choice of gauge will be to set, as is standard with points on $\mathbb{C P}^{1}$, the three points $z_{1}=\infty, z_{2}=1$ and $z_{n}=0$. Under this choice, the set of polynomial is reduced to

$$
\widetilde{h}_{1 \leq m \leq n-3} \equiv \lim _{z_{1} \rightarrow \infty} \frac{h_{m+1}}{z_{1}}=\left.\sum_{S \in A /\{1, n\},|S|=m}\left(k_{S}+k_{1}\right)^{2} z_{S}\right|_{z_{2}=1, z_{n}=0},
$$

In summary, $\tilde{h}$ defines a zero-dimensional ideal in the polynomial ring in $n-3$ variables. Then, using the standard Bézout's theorem, the number of points in this ideal (solutions of the scattering equation) is $\prod_{m=1}^{n-3} \operatorname{deg}\left(\widetilde{h}_{m}\right)=(n-3)$ !.

Instead of computing the amplitude with formula (2.6) by summing over all solutions of scattering equations, we will show in next section that, using the companion-matrix method, we can compute the amplitude $\mathcal{A}_{n}=\sum_{\text {sol }} \frac{P}{Q}$ as the trace of certain matrix composed of so-called companion matrices $T_{z_{i}}$

$$
\mathcal{A}_{n}=\operatorname{Tr}\left(\left.\left.P^{\prime}\right|_{z_{i} \rightarrow T_{z_{i}}} Q^{\prime-1}\right|_{z_{i} \rightarrow T_{z_{i}}}\right),
$$

without the explicit solutions of scattering equations.

\section{The mathematical framework}

As mentioned in the introduction, it is expedient to consider the problem within the framework of ideal theory. Our problem is thus the following.

Problem: $\quad$ Let $I=\left\langle f_{i}\right\rangle$ be a zero-dimensional ideal in $R=\mathbb{C}\left[x_{1}, \ldots, x_{n}\right]$ generated by $f_{i=1,2, \ldots, k}\left(x_{1}, \ldots, x_{n}\right) \in R$ and let $r\left(x_{1}, \ldots, x_{n}\right)$ be an arbitrary rational function in the fraction field of $R$. Because $\operatorname{dim}_{\mathbb{C}} I=0, I=\sqcup_{j=1}^{N}\left\{z_{j}\right\}$ is a discrete set of, say $N$, points. We wish to evaluate

$$
\sum_{j=1}^{N} p\left(z_{j}\right)
$$

where each summand is an evaluation of $p$ at one of the discrete set of zeros $z_{j}$. In particular we wish to test whether this sum is 0 . This is the level 3 equivalence mentioned in the introduction.

Of course, the idea is to solve this without explicitly finding the roots $z_{j}$. This can be done using the technique of companion matrices [40] (cf. also [41]). Suppose a Gröbner basis for $I$ has been found for some appropriate monomial ordering and B is an associated monomial basis for $I$, which can be seen as a vector space of dimension $d$. Then the multiplication map by the coordinate variable $x_{i}$

$$
\begin{aligned}
R / I & \longrightarrow R / I \\
T_{i}: \quad f & \longrightarrow x_{i} f
\end{aligned}
$$


is an endomorphism of quotient rings. In the basis $\mathrm{B}$ of monomials, this is a $d \times d$ matrix and is called a companion matrix. Clearly, $\left\{T_{i}\right\}$ all mutually commute and thus can be simultaneously diagonalized. We have the following [39]:

Theorem 3.0 (Stickelberger) The complex roots $z_{i}$ of I are the vectors of simultaneous eigenvalues of the companion matrices $T_{i=1, \ldots, n}$, i.e., the corresponding zero dimensional variety consists of the points:

$$
\mathcal{V}(I)=\left\{\left(\lambda_{1}, \ldots, \lambda_{n}\right) \in \mathbb{C}^{n}: \exists v \in \mathbb{C}^{n} \forall i: T_{i} v=\lambda_{i} v\right\} .
$$

We point out that the original statement of the theorem is in terms of annihilators in algebraic number theory and is perhaps a little abstruse. Fortunately, the computational algebraic-geometry community has rephrased this into the readily usable form of companion matrices [40, 42]. In particular, we have the following important consequence:

Corollary 1 Our desired quantity

$$
\sum_{j=1}^{N} r\left(z_{j}\right)=\operatorname{Tr}\left[r\left(T_{1}, \ldots, T_{n}\right)\right]
$$

where the evaluation of the rational function $r$ on the matrices $T_{i}$ is without ambiguity since they mutually commute.

We remark that because $r$ is rational, whenever the companion matrices appear in the denominator, they are to be understood as the inverse matrix.

\subsection{Warmup}

Before proceeding to examples in our context, we present two simple exercises to demonstrate our algorithm. Computations can be made in Macaulay2 [42] or Singular [43], or the latter's interface with Mathematica [44]. Let

$$
I:=\langle x y-z, y z-x, z x-y\rangle \subset R=\mathbb{C}[x, y, z] .
$$

We know, of course, that there are 5 roots

$$
\mathcal{V}(I)=\{(0,0,0),(-1,-1,1),(-1,1,-1),(1,-1,-1),(1,1,1)\} .
$$

Now we consider two functions, where one is polynomial and another, rational:

$$
p(x, y, z)=3 x^{3} y+x y z, \quad Q(x, y, z)=\frac{3 x^{3} y+x y z}{2 x y^{2}+4 z^{2}+1} .
$$

It is easy to find, after summing over the solutions, that

$$
\sum_{\mathcal{V}(I)} p=4, \quad \sum_{\mathcal{V}(I)} Q=\frac{20}{21} .
$$

We now show how the companion matrices work without finding the roots (3.3) explicitly. 
In the lex ordering of $x \prec y \prec z$, the Gröbner basis and the monomial basis are, respectively,

$$
G B(I)=\left\langle z^{3}-z, y z^{2}-y, y^{2}-z^{2}, x-y z\right\rangle ; \quad \mathrm{B}=\left\{1, y, y z, z, z^{2}\right\} .
$$

Therefore, we have that, in the quotient ring $R / I$,

$$
x . \mathrm{B}=\left\{y z, z, z^{2}, y, y z\right\}, \quad y \cdot \mathrm{B}=\left\{y, z^{2}, z, y z, y\right\}, \quad z . \mathrm{B}=\left\{z, y z, y, z^{2}, z\right\},
$$

so that

$$
T_{x}=\left(\begin{array}{lllll}
0 & 0 & 1 & 0 & 0 \\
0 & 0 & 0 & 1 & 0 \\
0 & 0 & 0 & 0 & 1 \\
0 & 1 & 0 & 0 & 0 \\
0 & 0 & 1 & 0 & 0
\end{array}\right), \quad T_{y}=\left(\begin{array}{ccccc}
0 & 1 & 0 & 0 & 0 \\
0 & 0 & 0 & 0 & 1 \\
0 & 0 & 0 & 1 & 0 \\
0 & 0 & 1 & 0 & 0 \\
0 & 1 & 0 & 0 & 0
\end{array}\right), \quad T_{z}=\left(\begin{array}{lllll}
0 & 0 & 0 & 1 & 0 \\
0 & 0 & 1 & 0 & 0 \\
0 & 1 & 0 & 0 & 0 \\
0 & 0 & 0 & 0 & 1 \\
0 & 0 & 0 & 1 & 0
\end{array}\right)
$$

Therefore, the sum over the roots of $p$ is

$$
\operatorname{Tr}\left(3 T_{x}^{3} T_{y}+T_{x} T_{y} T_{z}\right)=4
$$

and we have nice agreement with (3.4).

For the $Q$, the numerator is $N=3 T_{x}^{3} T_{y}+T_{x} T_{y} T_{z}=\left(\begin{array}{lllll}0 & 0 & 0 & 3 & 1 \\ 0 & 1 & 3 & 0 & 0 \\ 0 & 3 & 1 & 0 & 0 \\ 0 & 0 & 0 & 1 & 3 \\ 0 & 0 & 0 & 3 & 1\end{array}\right)$, while the denominator is $D=2 T_{x} T_{y}^{2}+4 T_{z}^{2}+I=\left(\begin{array}{lllll}1 & 0 & 2 & 0 & 4 \\ 0 & 5 & 0 & 2 & 0 \\ 0 & 0 & 5 & 0 & 2 \\ 0 & 2 & 0 & 5 & 0 \\ 0 & 0 & 2 & 0 & 5\end{array}\right)$. Thus we calculate $\operatorname{Tr}\left(N D^{-1}\right)=\frac{20}{21}$, which is the right answer on comparing with (3.4).

Before going to examples of scattering equations, let us give some remarks. First, the theorem in its original form is for polynomial test functions $r$, while functions we will meet in scattering equations are rational functions, i.e., the form $\frac{P}{Q}$ with both $P, Q$ are polynomials. Luckily, the theorem and corollary can be generalized trivially since we can diagonalize companion matrices simultaneously because the next remark.

Now, there is a second part of the theorem which states that the companion matrices can be simultaneously diagonalized if and only if the ideal $I$ is a radical ideal. That is, there are no multiple roots. However, as shown in [15], if all $k_{S}^{2} \neq 0$, the solutions of $z_{i}$ will all be different, so we indeed have a radical ideal and find simultaneous eigenvalues readily.

Third, since there are $(n-3)$ ! solutions, the size of $T_{i}$ will be in general $d=(n-3)$ ! which will become very large with $n$. Although with this counting, the efficiency of the method may be arguable, it does make the following property manifest: after summing over all solutions, the final result must be rational functions of $k, \epsilon$.

\section{Illustrative examples}

In the following, we will use several examples to demonstrate the companion matrix method. The $n=4$ case is simple. The companion matrix is 1-dimensional, equaling to the single solution of scattering equations. We compute the amplitudes in scalar $\phi^{3}$, 
Yang-Mills and gravity theories to show the validity of the method. For $n=5$, we first study the amplitude of scalar $\phi^{3}$ theory, and show that the amplitude-level identity can be understood by the fact that the trace of matrix is a linear mapping, and use it the explain a 7-point identity proposed in [31]. For the amplitudes of Yang-Mills and gravity theories, we will show that the companion matrix method indeed produce the correct amplitudes.

For $n=6$, the scalar $\phi^{3}$ theory will be shown to detect the pole structures so that the amplitude can be constructed by setting appropriate kinematics. The Next-MHV gluon amplitude is also presented as an example to show the validation of companion matrix method in a more difficult situation. Finally, for $n=7$ amplitudes of scalar $\phi^{3}$ theory, we demonstrate that, when companion matrices are computed in the diagonal form, the diagonal elements of the integrand matrix (which we recall to be an $(n-3) ! \times(n-3)$ ! matrix for $n$-points) have one-to-one mapping to the integrand computed at the $(n-3)$ ! solutions of the scattering equations, so they are not only equivalent at the amplitude level, but also at the level of each solution as indicated by Stickelberger's theorem.

\subsection{Four-point amplitudes}

The $n=4$ case is trivial. There is only $4-3=1$ variable left, so the companion matrix is just a complex number. Let us remove three scattering equations $\mathcal{E}_{1}, \mathcal{E}_{2}, \mathcal{E}_{4}$ and gauge-fix three points $z_{1}=\infty, z_{2}=1$ and $z_{4}=0$. The remaining one scattering equation is

$$
\mathcal{E}_{3}=\sum_{b \neq 3} \frac{s_{3 b}}{z_{3}-z_{b}}=\frac{s_{13}}{z_{3}-z_{1}}+\frac{s_{23}}{z_{3}-z_{2}}+\frac{s_{34}}{z_{3}-z_{4}}=\frac{\left(s_{23}+s_{34}\right) z_{3}-s_{34}}{z_{3}\left(z_{3}-1\right)}
$$

We can define the ideal $I=\left\langle\left(s_{23}+s_{34}\right) z_{3}-s_{34}\right\rangle$ in $\mathbb{C}\left[z_{3}\right]$. It is a linear function, so the Gröbner basis and monomial basis are trivially

$$
\mathrm{GB}(I)=\left\langle\left(s_{23}+s_{34}\right) z_{3}-s_{34}\right\rangle, \quad \mathrm{B}=\{1\} .
$$

The polynomial reduction of $z_{3} \mathrm{~B}=\left\{z_{3}\right\}$ over Gröbner basis of ideal $I$ gives the remainder $\left\{\frac{s_{34}}{s_{23}+s_{34}}\right\}$. Thus in the quotient ring, the companion matrix is given by

$$
T_{z_{3}} \mathrm{~B}=\left\{\frac{s_{34}}{s_{23}+s_{34}}\right\} \rightarrow T_{z_{3}}=\frac{s_{34}}{s_{23}+s_{34}} .
$$

We now proceed to the three cases of concern.

\subsubsection{Scalar $\phi^{3}$ theory}

For the 4-point amplitude in scalar $\phi^{3}$ theory, we wish to compute (recall that the three points $z_{1}, z_{2}, z_{4}$ have been gauge fixed)

$$
\mathcal{A}_{4}=\sum_{\text {sol }} \frac{z_{12}^{2} z_{24}^{2} z_{41}^{2}}{|\Phi|_{124}^{124}} \frac{1}{z_{12}^{2} z_{23}^{2} z_{34}^{2} z_{41}^{2}}=-\sum_{z_{3} \in \text { sol }} \frac{1}{s_{34}\left(z_{3}-1\right)^{2}+s_{23} z_{3}^{2}} \equiv \sum_{z_{3} \in \text { sol }} \frac{P\left(z_{3}\right)}{Q\left(z_{3}\right)},
$$

where we have used the simplification

$$
|\Phi|_{124}^{124}=\Phi_{33}=-\frac{s_{34}}{z_{3}^{2}}-\frac{s_{23}}{\left(z_{3}-1\right)^{2}} \rightarrow \frac{1}{|\Phi|_{124}^{124}}=-\frac{z_{3}^{2}\left(z_{3}-1\right)^{2}}{z_{3}^{2}\left(s_{12}+s_{23}\right)-2 z_{3} s_{12}+s_{12}},
$$


so that the factor $1 / z_{23}^{2} z_{34}^{2}$ cancels the numerator of $1 /|\Phi|_{124}^{124}$. We see that the final expression is summed over the (discrete) solution set of the scattering equation which is rather trivial here. The summand is a rational function in the free variable $z_{3}$ which we define as $P / Q$; of course, $P=1$ here and $Q$ will be used later.

Finally, using the simple expression for the companion matrix $T_{z_{3}}$ from (4.3), we have

$$
\operatorname{Tr}\left(\frac{P\left(T_{z_{3}}\right)}{Q\left(T_{z_{3}}\right)}\right)=\operatorname{Tr}\left(-\frac{1}{T_{z_{3}} T_{z_{3}}\left(s_{12}+s_{23}\right)-2 T_{z_{3}} s_{12}+s_{12}}\right)=-\frac{s_{23}+s_{34}}{s_{23} s_{34}}=-\frac{1}{s_{14}}-\frac{1}{s_{12}},
$$

after some identities between Mandelstam variables have been used. This is indeed the same answer as the standard known result as given in the introduction.

\subsubsection{Yang-Mills theory}

For 4-point amplitude in Yang-Mills theory, we want to compute (under gauge-fixing $z_{1}=$ $\left.\infty, z_{2}=1, z_{4}=0\right)$,

$$
\mathcal{A}_{4}^{\mathrm{YM}}=\sum_{\text {sol }} \frac{z_{12}^{2} z_{24}^{2} z_{41}^{2}}{|\Phi|_{124}^{124}} \frac{\mathrm{Pf}^{\prime} \Psi_{8 \times 8}}{z_{12} z_{23} z_{34} z_{41}} \equiv \sum_{z_{3} \in \text { sol }} \frac{P^{\mathrm{YM}}\left(z_{3}\right)}{Q^{\mathrm{YM}}\left(z_{3}\right)} .
$$

To avoid the divergence when taking the limit $z_{1} \rightarrow \infty$, one of the removed rows(columns) in $\Psi$ should be 1 , otherwise some terms in $\operatorname{Pf}^{\prime} \Psi_{8 \times 8}$ would lead to infinity. Let us then choose the reduced Pfaffian as

$$
\mathrm{Pf}^{\prime} \Psi_{8 \times 8}=\frac{-2}{z_{1}-z_{2}} \operatorname{Pf} \Psi_{12}^{12} .
$$

The large $z_{1}$ dependence of $\operatorname{Pf}^{\prime} \Psi_{8 \times 8}$ is then $1 / z_{1}^{2}$, and together with the factor from the scalar part, we obtain a finite integrand when taking the $z_{1} \rightarrow \infty$ limit. Explicitly, the new matrix $\widetilde{\Psi} \equiv \Psi_{12}^{12}$ is a $6 \times 6$ matrix,

$$
\widetilde{\Psi}=\left(\begin{array}{cccccc}
0 & \frac{k_{3} k_{4}}{z_{3}-z_{4}} & -\frac{\epsilon_{1} k_{3}}{z_{1}-z_{3}} & -\frac{\epsilon_{2} k_{3}}{z_{2}-z_{3}} & \sum_{c \neq 3} \frac{\epsilon_{3} k_{c}}{z_{3}-z_{c}} & -\frac{\epsilon_{4} k_{3}}{z_{4}-z_{3}} \\
\frac{k_{3} k_{4}}{z_{4}-z_{3}} & 0 & -\frac{\epsilon_{1} k_{4}}{z_{1}-z_{4}} & -\frac{\epsilon_{2} k_{4}}{z_{2}-z_{4}} & -\frac{\epsilon_{3} k_{4}}{z_{3}-z_{4}} & \sum_{c \neq 4} \frac{\epsilon_{4} k_{c}}{z_{4}-z_{c}} \\
\frac{\epsilon_{1} k_{3}}{z_{1}-z_{3}} & \frac{\epsilon_{1} k_{4}}{z_{1}-z_{4}} & 0 & \frac{\epsilon_{1} \epsilon_{2}}{z_{1}-z_{2}} & \frac{\epsilon_{1} \epsilon_{3}}{z_{1}-z_{3}} & \frac{\epsilon_{1} \epsilon_{4}}{z_{1}-z_{4}} \\
\frac{\epsilon_{2} k_{3}}{z_{2}-z_{3}} & \frac{\epsilon_{2} k_{4}}{z_{2}-z_{4}} & \frac{\epsilon_{1} \epsilon_{2}}{z_{2}-z_{1}} & 0 & \frac{\epsilon_{2} \epsilon_{3}}{z_{2}-z_{3}} & \frac{\epsilon_{2} \epsilon_{4}}{z_{2}-z_{4}} \\
-\sum_{c \neq 3} \frac{\epsilon_{3} k_{c}}{z_{3}-z_{c}} & \frac{\epsilon_{3} k_{4}}{z_{3}-z_{4}} & \frac{\epsilon_{1} \epsilon_{3}}{z_{3}-z_{1}} & \frac{\epsilon_{2} \epsilon_{3}}{z_{3}-z_{2}} & 0 & \frac{\epsilon_{3}}{z_{3}-z_{4}} \\
\frac{\epsilon_{4} k_{3}}{z_{4}-z_{3}} & -\sum_{c \neq 4} \frac{\epsilon_{4} k_{c}}{z_{4}-z_{c}} & \frac{\epsilon_{1} \epsilon_{4}}{z_{4}-z_{1}} & \frac{\epsilon_{2} \epsilon_{4}}{z_{4}-z_{2}} & \frac{\epsilon_{3} \epsilon_{4}}{z_{4}-z_{3}} & 0
\end{array}\right),
$$

whose Pfaffian is given by

$$
\begin{aligned}
\operatorname{Pf} \Psi_{12}^{12}= & \widetilde{\Psi}_{16} \widetilde{\Psi}_{25} \widetilde{\Psi}_{34}-\widetilde{\Psi}_{15} \widetilde{\Psi}_{26} \widetilde{\Psi}_{34}-\widetilde{\Psi}_{16} \widetilde{\Psi}_{24} \widetilde{\Psi}_{35}+\widetilde{\Psi}_{14} \widetilde{\Psi}_{26} \widetilde{\Psi}_{35}+\widetilde{\Psi}_{15} \widetilde{\Psi}_{24} \widetilde{\Psi}_{36} \\
& -\widetilde{\Psi}_{14} \widetilde{\Psi}_{25} \widetilde{\Psi}_{36}+\widetilde{\Psi}_{16} \widetilde{\Psi}_{23} \widetilde{\Psi}_{45}-\widetilde{\Psi}_{13} \widetilde{\Psi}_{26} \widetilde{\Psi}_{45}+\widetilde{\Psi}_{12} \widetilde{\Psi}_{36} \widetilde{\Psi}_{45}-\widetilde{\Psi}_{15} \widetilde{\Psi}_{23} \widetilde{\Psi}_{46} \\
& +\widetilde{\Psi}_{13} \widetilde{\Psi}_{25} \widetilde{\Psi}_{46}-\widetilde{\Psi}_{12} \widetilde{\Psi}_{35} \widetilde{\Psi}_{46}+\widetilde{\Psi}_{14} \widetilde{\Psi}_{23} \widetilde{\Psi}_{56}-\widetilde{\Psi}_{13} \widetilde{\Psi}_{24} \widetilde{\Psi}_{56}+\widetilde{\Psi}_{12} \widetilde{\Psi}_{34} \widetilde{\Psi}_{56}
\end{aligned}
$$

The reduced Pfaffian $\mathrm{Pf}^{\prime} \Psi_{8 \times 8}$ in this case is a rational function with denominator $z_{3}^{2}\left(z_{3}-1\right)$. Together with the factor $1 / z_{23} z_{34}=1 / z_{3}\left(z_{3}-1\right)$, they cancel the numerator of $1 /|\Phi|_{124}^{124}$, leaving a $z_{3}$ in the denominator of integrand. 
Therefore, it is immediate that the numerator of the integrand comes entirely from the numerator of the reduced Pfaffian:

$$
\begin{aligned}
P^{\mathrm{YM}}=z_{3}^{2}( & -s_{12} \widetilde{\epsilon}_{1,3} \widetilde{\epsilon}_{2,4}-2 \widetilde{\epsilon}_{3,4} \kappa_{1,3} \kappa_{2,4}+2 \widetilde{\epsilon}_{2,4} \kappa_{1,4} \kappa_{3,2}-2 \widetilde{\epsilon}_{1,4} \kappa_{2,4} \kappa_{3,2}+2 \widetilde{\epsilon}_{2,4} \kappa_{1,3} \kappa_{3,4} \\
& +2 \widetilde{\epsilon}_{2,4} \kappa_{1,4} \kappa_{3,4}-2 \widetilde{\epsilon}_{1,4} \kappa_{2,4} \kappa_{3,4}+2 \widetilde{\epsilon}_{2,3} \kappa_{1,3} \kappa_{4,2}-2 \widetilde{\epsilon}_{1,3} \kappa_{2,3} \kappa_{4,2}+2 \widetilde{\epsilon}_{1,2} \kappa_{3,2} \kappa_{4,2} \\
& \left.+2 \widetilde{\epsilon}_{1,2} \kappa_{3,4} \kappa_{4,2}+2 \widetilde{\epsilon}_{1,3} \kappa_{2,4} \kappa_{4,3}\right)+z_{3}\left(-s_{12} \widetilde{\epsilon}_{1,4} \widetilde{\epsilon}_{2,3}+s_{12} \widetilde{\epsilon}_{1,3} \widetilde{\epsilon}_{2,4}+s_{12} \widetilde{\epsilon}_{1,2} \widetilde{\epsilon}_{3,4}\right. \\
& -2 \widetilde{\epsilon}_{3,4} \kappa_{1,4} \kappa_{2,3}+2 \widetilde{\epsilon}_{3,4} \kappa_{1,3} \kappa_{2,4}-2 \widetilde{\epsilon}_{2,4} \kappa_{1,3} \kappa_{3,4}-2 \widetilde{\epsilon}_{2,4} \kappa_{1,4} \kappa_{3,4}+2 \widetilde{\epsilon}_{1,4} \kappa_{2,3} \kappa_{3,4} \\
& +2 \widetilde{\epsilon}_{1,4} \kappa_{2,4} \kappa_{3,4}-2 \widetilde{\epsilon}_{1,2} \kappa_{3,4} \kappa_{4,2}+2 \widetilde{\epsilon}_{2,3} \kappa_{1,3} \kappa_{4,3}+2 \widetilde{\epsilon}_{2,3} \kappa_{1,4} \kappa_{4,3}-2 \widetilde{\epsilon}_{1,3} \kappa_{2,3} \kappa_{4,3} \\
& \left.-2 \widetilde{\epsilon}_{1,3} \kappa_{2,4} \kappa_{4,3}+2 \widetilde{\epsilon}_{1,2} \kappa_{3,2} \kappa_{4,3}\right)-s_{12} \widetilde{\epsilon}_{1,2} \widetilde{\epsilon}_{3,4},
\end{aligned}
$$

where $\widetilde{\epsilon}_{i, j} \equiv \epsilon_{i} \epsilon_{j}, \kappa_{i, j} \equiv \epsilon_{i} k_{j}$. The denominator of the integrand, on the other hand, is

$$
Q^{\mathrm{YM}}=z_{3}^{3}\left(s_{12}+s_{23}\right)-2 z_{3}^{2} s_{12}+z_{3} s_{12}=z_{3} Q,
$$

where $Q$ is the denominator of integrand for the scalar $\phi^{3}$ theory from (4.4).

In summary, by computing $\operatorname{Tr}\left(\left.P Q^{-1}\right|_{z_{3} \rightarrow T_{z_{3}}}\right)$, we arrive at

$$
\begin{aligned}
\mathcal{A}_{4}^{\mathrm{YM}}=\widetilde{\epsilon}_{1,3} \widetilde{\epsilon}_{2,4} & -\widetilde{\epsilon}_{1,4} \widetilde{\epsilon}_{2,3}-\widetilde{\epsilon}_{1,2} \widetilde{\epsilon}_{3,4}-\frac{s_{12}}{s_{23}} \widetilde{\epsilon}_{1,4} \widetilde{\epsilon}_{2,3}-\frac{s_{23}}{s_{12}} \widetilde{\epsilon}_{1,2} \widetilde{\epsilon}_{3,4} \\
+\frac{1}{s_{12}}( & -2 \widetilde{\epsilon}_{3,4} \kappa_{1,4} \kappa_{2,3}+2 \widetilde{\epsilon}_{3,4} \kappa_{1,3} \kappa_{2,4}-2 \widetilde{\epsilon}_{2,4} \kappa_{1,3} \kappa_{3,4}-2 \widetilde{\epsilon}_{2,4} \kappa_{1,4} \kappa_{3,4}+2 \widetilde{\epsilon}_{1,4} \kappa_{2,3} \kappa_{3,4} \\
& +2 \widetilde{\epsilon}_{1,4} \kappa_{2,4} \kappa_{3,4}-2 \widetilde{\epsilon}_{1,2} \kappa_{3,4} \kappa_{4,2}+2 \widetilde{\epsilon}_{2,3} \kappa_{1,3} \kappa_{4,3}+2 \widetilde{\epsilon}_{2,3} \kappa_{1,4} \kappa_{4,3}-2 \widetilde{\epsilon}_{1,3} \kappa_{2,3} \kappa_{4,3} \\
& \left.-2 \widetilde{\epsilon}_{1,3} \kappa_{2,4} \kappa_{4,3}+2 \widetilde{\epsilon}_{1,2} \kappa_{3,2} \kappa_{4,3}\right) \\
+\frac{1}{s_{23}}( & -2 \widetilde{\epsilon}_{3,4} \kappa_{1,4} \kappa_{2,3}+2 \widetilde{\epsilon}_{2,4} \kappa_{1,4} \kappa_{3,2}-2 \widetilde{\epsilon}_{1,4} \kappa_{2,4} \kappa_{3,2}+2 \widetilde{\epsilon}_{1,4} \kappa_{2,3} \kappa_{3,4}+2 \widetilde{\epsilon}_{2,3} \kappa_{1,3} \kappa_{4,2} \\
& -2 \widetilde{\epsilon}_{1,3} \kappa_{2,3} \kappa_{4,2}+2 \widetilde{\epsilon}_{1,2} \kappa_{3,2} \kappa_{4,2}+2 \widetilde{\epsilon}_{2,3} \kappa_{1,3} \kappa_{4,3}+2 \widetilde{\epsilon}_{2,3} \kappa_{1,4} \kappa_{4,3}-2 \widetilde{\epsilon}_{1,3} \kappa_{2,3} \kappa_{4,3} \\
& \left.+2 \widetilde{\epsilon}_{1,2} \kappa_{3,2} \kappa_{4,3}\right) .
\end{aligned}
$$

The pole structures are similar to the scalar $\phi^{3}$ theory, while the terms without poles come from the gluon four-vertex. Of course, by momentum conservation and the property $\epsilon_{i} k_{i}=0$, we can further write the above result as a function of all independent kinematics, for example by using identities $\epsilon_{j} k_{4}=-\epsilon_{j} k_{3}-\epsilon_{j} k_{2}-\epsilon_{j} k_{1}$ and $\epsilon_{4} k_{4}=0$. This result agrees with the one computed directly by Feynman diagrams.

\subsubsection{Gravity}

For the 4-point amplitude in gravity, we want to compute

$$
\mathcal{A}_{4}^{\mathrm{G}}=\sum_{\text {sol }} \frac{z_{12}^{2} z_{24}^{2} z_{41}^{2}}{|\Phi|_{124}^{124}} \operatorname{Det}^{\prime}\left(\Psi_{8 \times 8}\right)=\sum_{\text {sol }} \frac{z_{12}^{2} z_{24}^{2} z_{41}^{2}}{|\Phi|_{124}^{124}}\left(\mathrm{Pf}^{\prime} \Psi_{8 \times 8}\right)^{2} \equiv \sum_{z_{3} \in \text { sol }} \frac{P^{\mathrm{G}}\left(z_{3}\right)}{Q^{\mathrm{G}}\left(z_{3}\right)},
$$

under the gauge-fixing $z_{1}=\infty, z_{2}=1, z_{4}=0$. As in Yang-Mills theory, we choose the reduced Pfaffian as

$$
\operatorname{Pf}^{\prime} \Psi_{8 \times 8}=\frac{-2}{z_{1}-z_{2}} \operatorname{Pf} \Psi_{12}^{12}
$$


and as above, we know that the squared reduced Pfaffian $\left(\operatorname{Pf}^{\prime} \Psi_{8 \times 8}\right)^{2}$ is a rational function with denominator $z_{3}^{4}\left(z_{3}-1\right)^{2}$. This cancels the numerator of $1 /|\Phi|_{124}^{124}$, leaving a $z_{3}^{2}$ in the denominator of integrand, so that the numerator of integrand equals to the square of numerator of reduced Pfaffian:

$$
P^{\mathrm{G}}=\left(P^{\mathrm{YM}}\right)^{2},
$$

while the denominator of integrand is

$$
Q^{\mathrm{G}}=z_{3}^{4}\left(s_{12}+s_{23}\right)-2 z_{3}^{3} s_{12}+z_{3}^{2} s_{12}=z_{3}^{2} Q=z_{3} Q^{\mathrm{YM}} .
$$

Combining all together, we have

$$
\frac{P^{\mathrm{G}}}{Q^{\mathrm{G}}}=\frac{\left(P^{\mathrm{YM}}\right)^{2}}{z_{3} Q^{\mathrm{YM}}}=\frac{Q^{\mathrm{YM}}}{z_{3}} \frac{\left(P^{\mathrm{YM}}\right)^{2}}{\left(Q^{\mathrm{YM}}\right)^{2}}=Q \frac{\left(P^{\mathrm{YM}}\right)^{2}}{\left(Q^{\mathrm{YM}}\right)^{2}},
$$

where $Q$ is the denominator of integrand for scalar $\phi^{3}$ theory from (4.4) and the expressions for $P^{\mathrm{YM}}$ and $Q^{\mathrm{YM}}$ are given in (4.11) and (4.12). In the present case of $n=4$, there is only one solution for scattering equations, and the companion matrix is really 1-dimensional in (4.3), so although in general $\operatorname{Tr}\left(M_{1} M_{2}\right) \neq \operatorname{Tr}\left(M_{1}\right) \operatorname{Tr}\left(M_{2}\right)$, here we simply have

$$
\operatorname{Tr}\left(\frac{P^{\mathrm{G}}}{Q^{\mathrm{G}}}\right)=\operatorname{Tr}(Q) \operatorname{Tr}\left(\frac{P^{\mathrm{YM}}}{Q^{\mathrm{YM}}}\right)^{2}=-\frac{s_{12} s_{23}}{s_{12}+s_{23}}\left(\mathcal{A}^{\mathrm{YM}}\right)^{2} .
$$

By BCJ relation [45], we can rewrite this to the familiar one

$$
\mathcal{A}_{4}^{\mathrm{G}}=s_{12} \mathcal{A}_{4}^{\mathrm{YM}}(1,2,3,4) \mathcal{A}_{4}^{\mathrm{YM}}(1,2,4,3),
$$

in agreement with the known result by KLT relation [46-50].

\subsection{Five-point amplitudes}

For $n=5$ amplitudes, there are five scattering equations, but only two of them are independent. Under the gauge-fixing $z_{1}=\infty, z_{2}=1, z_{5}=0$, the Dolan-Goddard's formula [15] gives:

$$
f_{1}=s_{12}+s_{13} z_{3}+s_{14} z_{4}, \quad f_{2}=s_{45} z_{3}+s_{35} z_{4}+s_{25} z_{3} z_{4} .
$$

We can solve these two equations to get two solutions:

$$
\operatorname{sol}_{1}: \quad z_{3}=\frac{-s_{12} s_{25}-s_{13} s_{35}+s_{14} s_{45}-\sqrt{\Delta}}{2 s_{13} s_{25}}, \quad z_{4}=\frac{-s_{12} s_{25}+s_{13} s_{35}-s_{14} s_{45}+\sqrt{\Delta}}{2 s_{14} s_{25}},
$$

and

$\operatorname{sol}_{2}: \quad z_{3}=\frac{-s_{12} s_{25}-s_{13} s_{35}+s_{14} s_{45}+\sqrt{\Delta}}{2 s_{13} s_{25}}, \quad z_{4}=\frac{-s_{12} s_{25}+s_{13} s_{35}-s_{14} s_{45}-\sqrt{\Delta}}{2 s_{14} s_{25}}$,

where $\Delta=\left(s_{12} s_{25}+s_{13} s_{35}-s_{14} s_{45}\right)^{2}-4 s_{12} s_{13} s_{25} s_{35}$. We can see that, in general the solutions are not rational functions, as is to be expected from high degree polynomials, 
though of course the final result of the sum over these points will be. One can see that the cancelations and simplifications will be very involved.

Let us turn to our companion matrix method. Define ideal $I=\left\langle f_{1}, f_{2}\right\rangle$ in the polynomial ring $\mathbb{C}\left[z_{3}, z_{4}\right]$, the Gröbner basis of ideal $I$ in Lexicographic order $z_{3} \prec z_{4}$ is given by

$$
\begin{aligned}
\mathrm{GB}(I)= & \left\langle s_{12} s_{45}+s_{12} s_{25} z_{4}-s_{13} s_{35} z_{4}+s_{14} s_{45} z_{4}+s_{14} s_{25} z_{4}^{2},\right. \\
& \left.s_{12}+s_{13} z_{3}+s_{14} z_{4}, \quad s_{45} z_{3}+s_{35} z_{4}+s_{25} z_{3} z_{4}\right\rangle .
\end{aligned}
$$

The monomial basis in this Gröbner basis is $\mathrm{B}=\left\{1, z_{4}\right\}$. Polynomial reduction of $z_{3} \mathrm{~B}$ and $z_{4} \mathrm{~B}$ over $\mathrm{GB}(I)$ gives the companion matrices $T_{z_{3}} \mathrm{~B}=z_{3} \mathrm{~B}, T_{z_{4}} \mathrm{~B}=z_{4} \mathrm{~B}$ as

$$
T_{z_{3}}=\left(\begin{array}{cc}
-\frac{s_{12}}{s_{13}} & -\frac{s_{14}}{s_{13}} \\
\frac{s_{12} s_{45}}{s_{13} s_{25}} & \frac{s_{14} s_{45}-s_{13} s_{35}}{s_{13} s_{25}}
\end{array}\right), \quad T_{z_{4}}=\left(\begin{array}{cc}
0 & 1 \\
-\frac{s_{12} s_{45}}{s_{14} s_{25}} & \frac{s_{13} s_{35}-s_{14} s_{45}-s_{12} s_{25}}{s_{14} s_{25}}
\end{array}\right)
$$

which are $2 \times 2$ matrices, in accordance with the number of solutions of scattering equations.

We note that the companion matrices actually formally "live" in the ideal $I$ itself by satisfying scattering equations, i.e.,

$$
\begin{aligned}
f_{1} & \rightarrow s_{12} I_{2 \times 2}+s_{13} T_{z_{3}}+s_{14} T_{z_{4}} \\
& =\left(\begin{array}{cc}
s_{12} & 0 \\
0 & s_{12}
\end{array}\right)+\left(\begin{array}{cc}
-s_{12} & -s_{14} \\
\frac{s_{12} s_{45}}{s_{25}} & \frac{s_{14} s_{45}-s_{13} s_{35}}{s_{25}}
\end{array}\right)+\left(\begin{array}{cc}
0 & s_{14} \\
-\frac{s_{12} s_{45}}{s_{25}} & \frac{s_{13} s_{35}-s_{14} s_{45}-s_{12} s_{25}}{s_{25}}
\end{array}\right)=0_{2 \times 2},
\end{aligned}
$$

and likewise, $s_{45} T_{z_{3}}+s_{35} T_{z_{4}}+s_{25} T_{z_{3}} T_{z_{4}}=0_{2 \times 2}$. This is, of course, a general property by construction since the companion matrices are constructed as multiplication (on a particular basis), so that substituting into the defining polynomials would vanish in the quotient ring. The situation is very much analogous to the classical result of Cayley-Hamilton that a matrix satisfies its own characteristic polynomial. It is worth to emphasize this discussion as

Corollary 2 The companion matrices satisfy the defining polynomials of the given ideal.

The above corollary shows some kind of equivalence between solutions of scattering equations and companion matrices of monomial basis over the Gröbner basis of scattering equations. With these companion matrices, we now proceed to compute the trace of the integrands to obtain the amplitude for different theories.

\subsubsection{Scalar $\phi^{3}$ theory}

The 5-point amplitude of scalar $\phi^{3}$ theory is given by

$$
\mathcal{A}_{5}=\sum_{\text {sol }} \frac{z_{12}^{2} z_{25}^{2} z_{51}^{2}}{|\Phi|_{125}^{125}} \frac{1}{z_{12}^{2} z_{23}^{2} z_{34}^{2} z_{45}^{2} z_{51}^{2}}=\sum_{\text {sol }} \frac{1}{|\Phi|_{125}^{125}\left(z_{3}-1\right)^{2}\left(z_{3}-z_{4}\right)^{2} z_{4}^{2}} \equiv \sum_{z_{3}, z_{4} \in \text { sol }} \frac{P\left(z_{3}, z_{4}\right)}{Q\left(z_{3}, z_{4}\right)},
$$

where we have used that

$$
|\Phi|_{125}^{125}=\left(-\frac{s_{23}}{\left(z_{3}-1\right)^{2}}-\frac{s_{34}}{\left(z_{3}-z_{4}\right)^{2}}-\frac{s_{35}}{z_{3}^{2}}\right)\left(-\frac{s_{24}}{\left(z_{4}-1\right)^{2}}-\frac{s_{34}}{\left(z_{3}-z_{4}\right)^{2}}-\frac{s_{45}}{z_{4}^{2}}\right)-\frac{s_{34}^{2}}{\left(z_{3}-z_{4}\right)^{4}}
$$


and as above, defined the appropriate $P$ and $Q$, which are, explicitly,

$$
\begin{aligned}
P= & z_{3}^{2}\left(z_{4}-1\right)^{2}, \\
Q= & \left(s_{35}\left(z_{3}-1\right)^{2}+s_{23} z_{3}^{2}\right)\left(z_{3}-z_{4}\right)^{2}\left(s_{45}\left(z_{4}-1\right)^{2}+s_{24} z_{4}^{2}\right) \\
& +s_{34}\left[s_{45}\left(z_{3}-1\right)^{2} z_{3}^{2}\left(z_{4}-1\right)^{2}\right. \\
& \left.+z_{4}^{2}\left(z_{3}^{2}\left(s_{24}\left(z_{3}-1\right)^{2}+s_{23}\left(z_{4}-1\right)^{2}\right)+s_{35}\left(z_{3}-1\right)^{2}\left(z_{4}-1\right)^{2}\right)\right] .
\end{aligned}
$$

Now, we wish to compute the trace of the matrix $P Q^{-1}$ upon substituting $z_{3}$ and $z_{4}$ by their associated companion matrices, instead of summing over all the complicated solutions of the scattering equations. In other words, we should replace the variables $z_{3}, z_{4}$ as $T_{z_{3}}, T_{z_{4}}$ in the integrand, i.e., $P^{\prime}=\left.P\right|_{z_{3} \rightarrow T_{z_{3}}, z_{4} \rightarrow T_{z_{4}}}, Q^{\prime}=\left.Q\right|_{z_{3} \rightarrow T_{z_{3}}, z_{4} \rightarrow T_{z_{4}}}$ (Hereafter we will always use $P^{\prime}, Q^{\prime}$ to denote the matrices after replacing $z_{i}$ to $T_{z_{i}}$ ). The product of variables $z_{3}, z_{4}$ changes to the product of matrices $T_{z_{3}}, T_{z_{4}}$, and since the companion matrices are commutable, their order does not matter in here. Then we should compute the inverse of matrix $Q^{\prime}$, and the final result is given by $\operatorname{Tr}\left(P^{\prime} Q^{\prime-1}\right)$.

Recalling that the physical poles appearing in the color-ordered amplitude are $s_{12}, s_{23}, s_{34}, s_{45}, s_{15}$, we can define them as the independent Mandelstam variables, and rewrite all the other Mandelstam variables in $P, Q, T_{z_{3}}, T_{z_{4}}$ by using following identities:

$$
\begin{array}{ll}
s_{35}=s_{12}-s_{34}-s_{45}, & s_{24}=s_{15}-s_{23}-s_{34}, \quad s_{25}=s_{34}-s_{15}-s_{12}, \\
s_{14}=s_{23}-s_{45}-s_{15}, & s_{13}=s_{45}-s_{12}-s_{23} .
\end{array}
$$

After some algebraic manipulation, readily performed by Mathematica, we obtain

$$
\operatorname{Tr}\left(P^{\prime}\left(T_{z_{3}}, T_{z_{4}}\right) Q^{\prime-1}\left(T_{z_{3}}, T_{z_{4}}\right)\right)=\frac{1}{s_{15} s_{23}}+\frac{1}{s_{12} s_{34}}+\frac{1}{s_{15} s_{34}}+\frac{1}{s_{12} s_{45}}+\frac{1}{s_{23} s_{45}},
$$

which agrees with the known result [30, 31].

Let us further consider an example, corresponding to the two-cycles ${ }^{3}$ $\{(1,2,3,4,5),(1,3,5,2,4)\}$, in the language of $[30,31,36]$. Using the CHY-integrand defined by above two-cycles, we have

$$
\mathcal{A}_{5}^{\prime}=\sum_{\text {sol }} \frac{z_{12}^{2} z_{25}^{2} z_{51}^{2}}{|\Phi|_{125}^{125}} \frac{1}{z_{12} z_{23} z_{34} z_{45} z_{51}} \frac{1}{z_{13} z_{35} z_{52} z_{24} z_{41}} \equiv \frac{P_{1}\left(z_{3}, z_{4}\right)}{Q_{1}\left(z_{3}, z_{4}\right)},
$$

which is represented by the so-called pentacle diagram (shown in figure 1)from the view of integration rules. Using the mapping rule given in [30,31], the answer is known to be zero. By directly computing the trace, we indeed find that $\operatorname{Tr}\left(P_{1}^{\prime} Q_{1}^{\prime-1}\right)=0$ and confirms this result.

In fact, for this example, although CHY-integrands of $\mathcal{A}_{5}$ and $\mathcal{A}_{5}^{\prime}$ are different, after simplification, their difference appears only in the numerator, i.e.,

$$
P_{1}=z_{3} z_{4}\left(1-z_{3}\right)\left(1-z_{4}\right)\left(z_{3}-z_{4}\right), \quad Q_{1}=Q .
$$

\footnotetext{
${ }^{3}$ Each cycle defines an expression, e.g., $\operatorname{Cycle}_{a}(1,3,5,2,4)=1 /\left(z_{13} z_{35} z_{52} z_{24} z_{41}\right)$, and the two-cycles denotes the expression given by $\mathrm{Cycle}_{a} \mathrm{Cycle}_{b}$.
} 


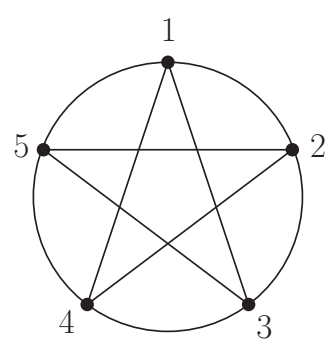

Figure 1. The pentacle diagram representing the CHY-integrand defined by the two-cycles $\{(1,2,3,4,5),(1,3,5,2,4)\}$.

Since the trace of matrix is a linear mapping, in particular $\operatorname{Tr}\left(M_{1}+M_{2}\right)=\operatorname{Tr}\left(M_{1}\right)+\operatorname{Tr}\left(M_{2}\right)$, relations between results of different integrands should also have hints in the integrand level. For example, let us consider the following three CHY-integrands defined by three two-cycles $\alpha_{2} \equiv\{(1,2,3,4,5),(1,2,3,5,4)\}, \alpha_{3} \equiv\{(1,2,3,4,5),(1,2,4,5,3)\}$ and $\alpha_{4} \equiv$ $\{(1,2,3,4,5),(1,3,2,5,4)\}$. With some calculations, we find

$$
\begin{aligned}
& \mathcal{A}_{5}\left(\alpha_{2}\right)=\sum_{\text {sol }} \frac{z_{12}^{2} z_{25}^{2} z_{51}^{2}}{|\Phi|_{125}^{125}} \frac{1}{z_{12} z_{23} z_{34} z_{45} z_{51}} \frac{1}{z_{12} z_{23} z_{35} z_{54} z_{41}} \equiv \sum_{\text {sol }} \frac{P_{2}}{Q} \\
& \mathcal{A}_{5}\left(\alpha_{3}\right)=\sum_{\text {sol }} \frac{z_{12}^{2} z_{25}^{2} z_{51}^{2}}{|\Phi|_{125}^{125}} \frac{1}{z_{12} z_{23} z_{34} z_{45} z_{51}} \frac{1}{z_{12} z_{24} z_{45} z_{53} z_{31}} \equiv \sum_{\text {sol }} \frac{P_{3}}{Q} \\
& \mathcal{A}_{5}\left(\alpha_{4}\right)=\sum_{\text {sol }} \frac{z_{12}^{2} z_{25}^{2} z_{51}^{2}}{|\Phi|_{125}^{25}} \frac{1}{z_{12} z_{23} z_{34} z_{45} z_{51}} \frac{1}{z_{13} z_{32} z_{25} z_{54} z_{41}} \equiv \sum_{\text {sol }} \frac{P_{4}}{Q}
\end{aligned}
$$

where they share the same denominator $Q$, but different numerators

$$
\begin{aligned}
& P_{2}=z_{3}\left(z_{3}-z_{4}\right)\left(z_{4}-1\right)^{2}, \quad P_{3}=z_{3}\left(z_{3}-1\right)\left(z_{3}-z_{4}\right)\left(z_{4}-1\right) \\
& P_{4}=z_{3}^{2}\left(z_{3}-z_{4}\right)\left(z_{4}-1\right)^{2}
\end{aligned}
$$

After putting back the companion matrices, we find that

$$
\begin{aligned}
& \mathcal{A}_{5}\left(\alpha_{2}\right)=\operatorname{Tr}\left(P_{2}^{\prime} Q^{\prime-1}\right)=\frac{1}{s_{12} s_{45}}+\frac{1}{s_{23} s_{45}}, \\
& \mathcal{A}_{5}\left(\alpha_{3}\right)=\operatorname{Tr}\left(P_{3}^{\prime} Q^{\prime-1}\right)=\frac{1}{s_{12} s_{45}}, \mathcal{A}_{5}\left(\alpha_{4}\right)=\operatorname{Tr}\left(P_{4}^{\prime} Q^{\prime-1}\right)=\frac{1}{s_{23} s_{45}} .
\end{aligned}
$$

Realizing that the polynomials have the simple relation

$$
P_{3}+P_{4}-P_{2}=P_{1}
$$

we obtain the identity amongst these amplitudes as

$$
\begin{aligned}
\operatorname{Tr}\left(P_{2}^{\prime} Q^{\prime-1}\right) & =\operatorname{Tr}\left(\left(P_{3}^{\prime}+P_{4}^{\prime}-P_{1}^{\prime}\right) Q^{\prime-1}\right)=\operatorname{Tr}\left(P_{3}^{\prime} Q^{\prime-1}\right)+\operatorname{Tr}\left(P_{4}^{\prime} Q^{\prime-1}\right)+\operatorname{Tr}\left(P_{1}^{\prime} Q^{\prime-1}\right) \\
\rightarrow \mathcal{A}_{5}\left(\alpha_{2}\right) & =\mathcal{A}_{5}\left(\alpha_{3}\right)+\mathcal{A}_{5}\left(\alpha_{4}\right)+0 .
\end{aligned}
$$


Above example demonstrates an idea how to find relations among different amplitudes. Starting from different CHY-integrands, we can equalize their denominators by multiplying proper polynomial both at the denominator and the numerator. After that, the relations among different amplitudes can be understood from the relations among different numerators.

Let us demonstrate above idea by another example, i.e., the 7-point amplitude-level identity given by eq. (3.7) of [31], viz., amplitude obtained from the CHY-integrand

$$
\frac{1}{z_{12} z_{23} z_{34} z_{45} z_{56} z_{67} z_{71}} \frac{1}{z_{12} z_{27} z_{74} z_{46} z_{65} z_{53} z_{31}}
$$

is identical to the sum of following two amplitudes obtained from two CHY-integrand

$$
\frac{1}{z_{12} z_{23} z_{34} z_{45} z_{56} z_{67} z_{71}} \frac{1}{z_{12} z_{56} z_{37} z_{46}}\left(\frac{1}{z_{14} z_{27} z_{35}}+\frac{1}{z_{25} z_{74} z_{31}}\right) \text {. }
$$

Under gauge-fixing $z_{1}=\infty, z_{2}=1, z_{7}=0$ and excluding the 1-st, 2-nd and 7-th scattering equations, the Jacobian is

$$
\frac{1}{|\Phi|_{127}^{127}}=\frac{\prod_{i=3}^{6} z_{i}^{2}\left(z_{i}-1\right)^{2} \prod_{3 \leq i<j \leq 6}\left(z_{i}-z_{j}\right)^{2}}{Q}
$$

Thus we can immediately get the numerator of integrand after inserting the above three terms. The first term gives

$$
P_{1}=z_{3} z_{5}\left(z_{4}-1\right)\left(z_{5}-1\right)\left(z_{6}-1\right)\left(z_{3}-z_{6}\right) \prod_{i=3}^{6} z_{i}\left(z_{i}-1\right) \prod_{3 \leq i<j \leq 6}^{i \neq 5}\left(z_{i}-z_{j}\right),
$$

while the other two terms give

$$
\begin{aligned}
& P_{2}=z_{4} z_{5}\left(z_{4}-1\right)\left(z_{5}-1\right)\left(z_{6}-1\right)\left(z_{3}-z_{6}\right) \prod_{i=3}^{6} z_{i}\left(z_{i}-1\right) \prod_{3 \leq i<j \leq 6}^{i \neq 5}\left(z_{i}-z_{j}\right), \\
& P_{3}=-z_{5}\left(z_{4}-1\right)\left(z_{6}-1\right)\left(z_{3}-z_{5}\right)\left(z_{3}-z_{6}\right) \prod_{i=3}^{6} z_{i}\left(z_{i}-1\right) \prod_{3 \leq i<j \leq 6}^{i \neq 5}\left(z_{i}-z_{j}\right) .
\end{aligned}
$$

Note that

$$
\begin{aligned}
& P_{1}-P_{2}-P_{3} \\
& =z_{5}\left(z_{4}-1\right)\left(z_{6}-1\right)\left(z_{3}-z_{6}\right)\left(z_{4}-z_{5}+z_{3} z_{5}-z_{4} z_{5}\right) \prod_{i=3}^{6} z_{i}\left(z_{i}-1\right) \prod_{3 \leq i<j \leq 6}^{i \neq 5}\left(z_{i}-z_{j}\right),
\end{aligned}
$$

while the trace $\operatorname{Tr}\left(\left(P_{1}^{\prime}-P_{2}^{\prime}-P_{3}^{\prime}\right) Q^{\prime-1}\right)$ is zero. Note also the following decomposition

$$
z_{4}-z_{5}+z_{3} z_{5}-z_{4} z_{5}=\left(z_{3}-1\right)\left(z_{5}-z_{4}\right)+z_{4}\left(z_{3}-z_{5}\right)
$$


so that we can write $P_{1}-P_{2}-P_{3}=P_{4}+P_{5}$, with

$$
\begin{aligned}
& P_{4}=-z_{5}\left(z_{3}-1\right)\left(z_{4}-1\right)\left(z_{6}-1\right)\left(z_{3}-z_{6}\right)\left(z_{4}-z_{5}\right) \prod_{i=3}^{6} z_{i}\left(z_{i}-1\right) \prod_{3 \leq i<j \leq 6}^{i \neq 5}\left(z_{i}-z_{j}\right), \\
& P_{5}=z_{4} z_{5}\left(z_{4}-1\right)\left(z_{6}-1\right)\left(z_{3}-z_{5}\right)\left(z_{3}-z_{6}\right) \prod_{i=3}^{6} z_{i}\left(z_{i}-1\right) \prod_{3 \leq i<j \leq 6}^{i \neq 5}\left(z_{i}-z_{j}\right),
\end{aligned}
$$

which correspond to two-cycles

$$
\{(1,2,7,4,6,5,3),(1,2,5,6,7,3,4)\}, \quad\{(1,2,3,4,5,6,7),(1,3,7,2,5,6,4)\}
$$

respectively with $\operatorname{Tr}\left(P_{4}^{\prime} Q^{\prime-1}\right)=0, \operatorname{Tr}\left(P_{5}^{\prime} Q^{\prime-1}\right)=0$.

We thus conclude that strictly speaking, the amplitude-level identity between (4.35) and (4.36) is up to some CHY-integrands which have vanishing amplitude. More explicitly, the identity $(4.35)=(4.36)+(4.45)$ holds exactly at the integrand-level, while (4.45) has vanishing final result, so that $(4.35)=(4.36)$ holds at the amplitude-level. This provides the amplitude-level identity an explanation from the basic linearity of the trace.

\subsubsection{Yang-Mills theory}

For 5-point amplitude in Yang-Mills theory, we want to compute

$$
\mathcal{A}_{5}^{\mathrm{YM}}=\sum_{\text {sol }} \frac{z_{12}^{2} z_{25}^{2} z_{51}^{2}}{|\Phi|_{125}^{25}} \frac{\mathrm{Pf}^{\prime} \Psi_{10 \times 10}}{z_{12} z_{23} z_{34} z_{45} z_{51}} \equiv \sum_{z_{3}, z_{4} \in \text { sol }} \frac{P^{\mathrm{YM}}\left(z_{3}, z_{4}\right)}{Q^{\mathrm{YM}}\left(z_{3}, z_{4}\right)},
$$

under gauge-fixing $z_{1}=\infty, z_{2}=1, z_{5}=0$. Let us choose the reduced Pfaffian as

$$
\operatorname{Pf}^{\prime} \Psi_{10 \times 10}=\frac{-2}{z_{1}-z_{2}} \operatorname{Pf} \Psi_{12}^{12}
$$

where $\Psi_{12}^{12}$ is a $8 \times 8$ matrix. As in the 4 -point case, the large $z_{1}$ dependence of $\operatorname{Pf}^{\prime} \Psi_{10 \times 10}$ is $1 / z_{1}^{2}$, while $1 /\left(z_{12} z_{23} z_{34} z_{45} z_{51}\right)$ is also $1 / z_{1}^{2}$. Together with the factor $z_{12}^{2} z_{25}^{2} z_{51}^{2}$ in numerator, we get a finite integrand under the $z_{1} \rightarrow \infty$ limit.

We now follow the standard computation procedure:

1. Write down the expressions for $|\Phi|_{125}^{125}$ and $\operatorname{Pf}^{\prime} \Psi_{10 \times 10}$, and work out $P^{\mathrm{YM}}\left(z_{3}, z_{4}\right)$, $Q^{\mathrm{YM}}\left(z_{3}, z_{4}\right)$

2. Replace the variables $z_{i}$ 's by companion matrices $T_{z_{i}}$, as $P^{\prime}=\left.P\right|_{z_{i} \rightarrow T_{z_{i}}}, Q^{\prime}=$ $\left.Q\right|_{z_{i} \rightarrow T_{z_{i}}}$

3. Compute the inverse of $Q^{\prime}$ and the trace $\operatorname{Tr}\left(P^{\prime} Q^{\prime-1}\right)$.

The result for un-specified helicities is quite lengthy. For illustration, let us consider the 5-point amplitude with helicity $\mathcal{A}_{5}^{\mathrm{YM}}\left(g_{1}^{-}, g_{2}^{-}, g_{3}^{+}, g_{4}^{+}, g_{5}^{+}\right)$. The polarization vector is defined as

$$
\epsilon_{\mu}^{-}(k)=\frac{\left\langle k\left|\gamma_{\mu}\right| r\right]}{\sqrt{2}[k r]}, \quad \epsilon_{\mu}^{+}(k)=\frac{\left\langle r\left|\gamma_{\mu}\right| k\right]}{\sqrt{2}\langle r k\rangle}
$$


and we choose the reference momenta as $r_{1}=r_{2}=k_{3}, r_{3}=r_{4}=r_{5}=k_{2}$. Thus settled, the only surviving products of polarization vectors are $\epsilon^{-}\left(k_{1}\right) \cdot \epsilon^{+}\left(k_{4}\right)$ and $\epsilon^{-}\left(k_{1}\right) \cdot \epsilon^{+}\left(k_{5}\right)$. After imposing momentum conservation for $\epsilon^{ \pm}\left(k_{i}\right) \cdot k_{j}$ to reduce the ambiguity, we can simplify the $8 \times 8$ matrix $\widetilde{\Psi} \equiv \Psi_{12}^{12}$ as

$$
\left(\begin{array}{cccccccc}
0 & \frac{k_{3} k_{4}}{z_{3}-z_{4}} & \frac{k_{3} k_{5}}{z_{3}-z_{5}} & 0 & 0 & \widetilde{\Psi}_{16} & -\frac{\epsilon_{4} k_{3}}{z_{4}-z_{3}} & -\frac{\epsilon_{5} k_{3}}{z_{5}-z_{3}} \\
\frac{k_{3} k_{4}}{z_{4}-z_{3}} & 0 & \frac{k_{4} k_{5}}{z_{4}-z_{5}} & -\frac{\epsilon_{1} k_{4}}{z_{1}-z_{4}} & -\frac{\epsilon_{2} k_{4}}{z_{2}-z_{4}} & -\frac{\epsilon_{3} k_{4}}{z_{3}-z_{4}} & \Psi_{27} & \frac{\epsilon_{5} k_{1}+\epsilon_{5} k_{3}}{z_{3}-z_{4}} \\
\frac{k_{3} k_{5}}{z_{5}-z_{3}} & \frac{k_{4} k_{5}}{z_{5}-z_{4}} & 0 & \frac{\epsilon_{1} k_{2}+\epsilon_{1} k_{4}}{z_{1}-z_{5}} & \frac{\epsilon_{2} k_{1}+\epsilon_{2} k_{4}}{z_{2}-z_{5}} & \frac{\epsilon_{3} k_{1}+\epsilon_{3} k_{4}}{z_{3}-z_{5}} & \frac{\epsilon_{4} k_{1}+\epsilon_{4} k_{3}}{z_{4}-z_{5}} & \Psi_{38} \\
0 & \frac{\epsilon_{1} k_{4}}{z_{1}-z_{4}} & -\frac{\epsilon_{1} k_{2}+\epsilon_{1} k_{4}}{z_{1}-z_{5}} & 0 & 0 & 0 & \frac{\epsilon_{1} \epsilon_{4}}{z_{1}-z_{4}} & \frac{\epsilon_{1} \epsilon_{5}}{z_{1}-z_{5}} \\
0 & \frac{\epsilon_{2} k_{4}}{z_{2}-z_{4}} & -\frac{\epsilon_{2} k_{1}+\epsilon_{2} k_{4}}{z_{2}-z_{5} k_{4}} & 0 & 0 & 0 & 0 & 0 \\
\widetilde{\Psi}_{61} & \frac{\epsilon_{3} k_{4}}{z_{3}-z_{4}} & -\frac{\epsilon_{3} k_{1}+\epsilon_{3} k_{4}}{z_{3}-z_{5} k_{3}} & 0 & 0 & 0 & 0 & 0 \\
\frac{\epsilon_{4} k_{3}}{z_{4}-z_{3}} & \Psi_{72} & -\frac{\epsilon_{4} k_{1}+\epsilon_{4} k_{3}}{z_{4}-z_{5}} & \frac{\epsilon_{1} \epsilon_{4}}{z_{4}-z_{1}} & 0 & 0 & 0 & 0 \\
\frac{\epsilon_{5} k_{3}}{z_{5}-z_{3}} & -\frac{\epsilon_{5} k_{1}+\epsilon_{5} k_{3}}{z_{5}-z_{4}} & \widetilde{\Psi}_{83} & \frac{\epsilon_{1} \epsilon_{5}}{z_{5}-z_{1}} & 0 & 0 & 0 & 0
\end{array}\right),
$$

where

$$
\begin{aligned}
& \widetilde{\Psi}_{16}=-\widetilde{\Psi}_{61}=\frac{\epsilon_{3} k_{1}}{z_{3}-z_{1}}+\frac{\epsilon_{3} k_{4}}{z_{3}-z_{4}}-\frac{\epsilon_{3} k_{1}+\epsilon_{3} k_{4}}{z_{3}-z_{5}}, \\
& \widetilde{\Psi}_{27}=-\widetilde{\Psi}_{72}=\frac{\epsilon_{4} k_{1}}{z_{4}-z_{1}}+\frac{\epsilon_{4} k_{3}}{z_{4}-z_{3}}-\frac{\epsilon_{4} k_{1}+\epsilon_{4} k_{3}}{z_{4}-z_{5}}, \\
& \widetilde{\Psi}_{38}=-\widetilde{\Psi}_{83}=\frac{\epsilon_{5} k_{1}}{z_{5}-z_{1}}+\frac{\epsilon_{5} k_{3}}{z_{5}-z_{3}}-\frac{\epsilon_{5} k_{1}+\epsilon_{5} k_{3}}{z_{5}-z_{4}} .
\end{aligned}
$$

This greatly simplifies the result of reduced Pfaffian, which reads, after our gauge-fixing,

$$
\begin{aligned}
\operatorname{Pf}^{\prime} \Psi_{10 \times 10} \equiv & \frac{N_{\Psi}}{D_{\Psi}}=-2 \frac{z_{4} \kappa_{2,1}+z_{4} \kappa_{2,4}-\kappa_{2,1}}{z_{3} z_{4}\left(z_{4}-1\right)\left(z_{3}-z_{4}\right)}\left(\left(z_{3}-z_{4}\right) \widetilde{\epsilon}_{1,5} \kappa_{3,1} \kappa_{4,1}-\left(z_{3}-z_{4}\right) \widetilde{\epsilon}_{1,4} \kappa_{3,1} \kappa_{5,1}\right. \\
& \left.-\left(z_{3}-z_{4}\right) \widetilde{\epsilon}_{1,4} \kappa_{3,1} \kappa_{5,3}-z_{4} \widetilde{\epsilon}_{1,5} \kappa_{3,4} \kappa_{4,1}+z_{3} \widetilde{\epsilon}_{1,5} \kappa_{3,1} \kappa_{4,3}+z_{4} \widetilde{\epsilon}_{1,4} \kappa_{3,4} \kappa_{5,1}\right),(4.51
\end{aligned}
$$

where we recall again that $\widetilde{\epsilon}_{i, j}=\epsilon_{i} \epsilon_{j}, \kappa_{i, j}=\epsilon_{i} k_{j}$. The factor of scalar part $1 /\left(z_{12} z_{23} z_{34} z_{45} z_{51}\right)$ after gauge fixing is $\frac{1}{z_{4}\left(z_{3}-1\right)\left(z_{3}-z_{4}\right)}$, and the Jacobian $|\Phi|_{125}^{125}$ is the same as in the scalar theory,

$$
\frac{1}{|\Phi|_{125}^{125}}=\frac{z_{3}^{2} z_{4}^{2}\left(z_{3}-1\right)^{2}\left(z_{4}-1\right)^{2}\left(z_{3}-z_{4}\right)^{2}}{Q^{\mathrm{YM}}}
$$

where $Q^{\mathrm{YM}}$ is a polynomial of $z_{3}, z_{4}$ and Mandelstam variables, and it is also the denominator of integrand. The numerator of $1 /|\Phi|_{125}^{125}$ cancels the denominator of $\operatorname{Pf}^{\prime} \Psi$ and that of scalar part, leaving a factor $z_{3}\left(z_{3}-1\right)\left(z_{4}-1\right)$ in the numerator. Combined with the numerator $N_{\Psi}$ of $\operatorname{Pf}^{\prime} \Psi_{10 \times 10}$, they contribute to $P^{\mathrm{YM}}=z_{3}\left(z_{3}-1\right)\left(z_{4}-1\right) N_{\Psi}$.

Then it is straightforward to apply the replacements $P^{/ \mathrm{YM}}\left(T_{z_{3}}, T_{z_{4}}\right)=$ $\left.P^{\mathrm{YM}}\left(z_{3}, z_{4}\right)\right|_{z_{i} \rightarrow T_{z_{i}}}, \quad Q^{\prime \mathrm{YM}}\left(T_{z_{3}}, T_{z_{4}}\right)=\left.Q^{\mathrm{YM}}\left(z_{3}, z_{4}\right)\right|_{z_{i} \rightarrow T_{z_{i}}}$, and compute the trace $\operatorname{Tr}\left(P^{\prime Y M}\left(Q^{\prime Y M}\right)^{-1}\right)$. To make the computation more efficient, we can firstly apply the polynomial reduction of $P^{\mathrm{YM}}\left(z_{3}, z_{4}\right), Q^{\mathrm{YM}}\left(z_{3}, z_{4}\right)$ over $\operatorname{GB}(I)$. The remainders $R\left(P^{\mathrm{YM}}\right), R\left(Q^{\mathrm{YM}}\right)$ are polynomials of $z_{4}$ only, since the monomial basis is $\left\{1, z_{4}\right\}$. Then we can proceed by replacing $z_{4} \rightarrow T_{z_{4}}$ for the remainders, and compute the corresponding trace. This gives 
the same result as with the original $P^{\mathrm{YM}}, Q^{\mathrm{YM}}$, but the computation would be much faster. With Mathematica, we obtain

$$
\begin{aligned}
\operatorname{Tr}\left(P^{\prime \mathrm{YM}}\left(Q^{\prime \mathrm{YM}}\right)^{-1}\right)= & 2 \frac{\widetilde{\epsilon}_{1,5} \kappa_{2,1} \kappa_{3,1} \kappa_{4,3}+\widetilde{\epsilon}_{1,4} \kappa_{2,1} \kappa_{3,4} \kappa_{5,1}-\widetilde{\epsilon}_{1,5} \kappa_{2,1} \kappa_{3,4} \kappa_{4,1}}{s_{12} s_{34}} \\
& +2 \frac{\widetilde{\epsilon}_{1,5} \kappa_{2,1} \kappa_{3,1} \kappa_{4,1}+\widetilde{\epsilon}_{1,5} \kappa_{2,1} \kappa_{3,1} \kappa_{4,3}-\widetilde{\epsilon}_{1,4} \kappa_{2,1} \kappa_{3,1} \kappa_{5,1}-\widetilde{\epsilon}_{1,4} \kappa_{2,1} \kappa_{3,1} \kappa_{5,3}}{s_{12} s_{45}} \\
& +2 \frac{\widetilde{\epsilon}_{1,5} \kappa_{2,4} \kappa_{3,4} \kappa_{4,1}-\widetilde{\epsilon}_{1,5} \kappa_{2,4} \kappa_{3,1} \kappa_{4,3}-\widetilde{\epsilon}_{1,4} \kappa_{2,4} \kappa_{3,4} \kappa_{5,1}}{s_{15} s_{34}} .
\end{aligned}
$$

The missing of the pole terms $1 /\left(s_{15} s_{23}\right), 1 /\left(s_{23} s_{45}\right)$ (terms involving pole $\left.s_{23}\right)$ is due to the choice of polarization vectors. However, the $s_{23}$ pole do exist, hiding in $\kappa_{2, i}, \kappa_{3, i}$. Directly rewriting the spinor brackets for $\widetilde{\epsilon}_{i, j}, \kappa_{i, j}$ and $s_{i j}$, and using the Schouten identities we get the famous MHV-amplitude [51, 52]

$$
\operatorname{Tr}\left(P^{\prime \mathrm{YM}}\left(Q^{\prime \mathrm{YM}}\right)^{-1}\right)=\frac{\langle 12\rangle^{4}}{\langle 12\rangle\langle 23\rangle\langle 34\rangle\langle 45\rangle\langle 51\rangle} .
$$

\subsubsection{Gravity and $n$-point KLT relations}

For 5-point amplitude in pure gravity theory, under gauge-fixing $z_{1}=\infty, z_{2}=1, z_{5}=0$, we wish to compute

$$
\mathcal{A}_{5}^{\mathrm{G}}=\sum_{\text {sol }} \frac{z_{12}^{2} z_{25}^{2} z_{51}^{2}}{|\Phi|_{125}^{125}}\left(\mathrm{Pf}^{\prime} \Psi_{10 \times 10}\right)\left(\mathrm{Pf}^{\prime} \widetilde{\Psi}_{10 \times 10}\right)=\sum_{\text {sol }} \frac{z_{12}^{2} z_{25}^{2} z_{51}^{2}}{|\Phi|_{125}^{125}}\left(\mathrm{Pf}^{\prime} \Psi_{10 \times 10}\right)^{2} .
$$

Let us consider the gravity amplitude $\mathcal{A}_{5}^{\mathrm{G}}\left(1^{--}, 2^{--}, 3^{++}, 4^{++}, 5^{++}\right)$, so that we can use the same reduced Pfaffian $\mathrm{Pf}^{\prime} \Psi_{10 \times 10}$ as in the Yang-Mills case. Here, we do not have the factor of scalar part, but the square of the factor of the reduced Pfaffian. The numerator of $1 /|\Phi|_{125}^{125}$ cancels the squared denominator of reduced Pfaffian $z_{3}^{2} z_{4}^{2}\left(z_{4}-1\right)^{2}\left(z_{3}-z_{4}\right)^{2}$, leaving a factor of $\left(z_{3}-1\right)^{2}$ in the numerator. Hence, we have $Q^{\mathrm{G}}=Q^{\mathrm{YM}}$, and $P^{\mathrm{G}}=\left(z_{3}-1\right)^{2} N_{\Psi}^{2}$ with $N_{\Psi}$ given in (4.51).

Thus, all the ingredients have been computed in the Yang-Mills situation above, and we only need to work out the trace $\operatorname{Tr}\left(P^{\prime \mathrm{G}}\left(Q^{\prime \mathrm{G}}\right)^{-1}\right)$, which gives a lengthy result:

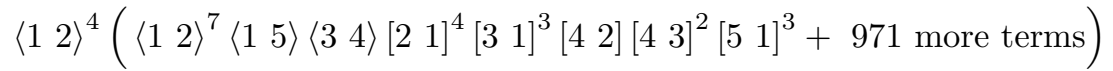

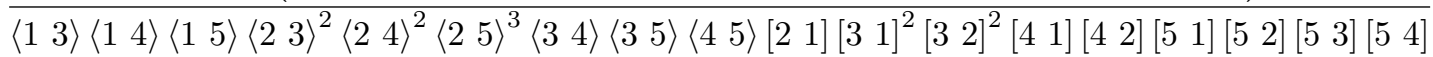

where we can see that all poles $s_{i, j}, i, j=1, \ldots, 5$ appearing therein, indicating the colorless structure of gravity amplitude.

This complicated expression can be simplified by non-trivially imposing momentum conservation and Schouten identities. Applying the algorithm described in the appendix of [53], for instance, we can simplify $A_{5}^{\mathrm{G}}\left(1^{--}, 2^{--}, 3^{++}, 4^{++}, 5^{++}\right)$to

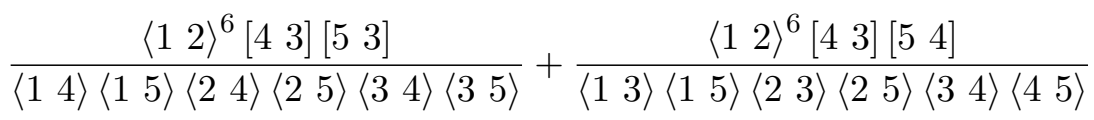

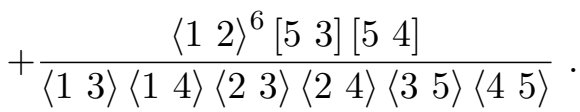

which agrees perfectly with the result given by KLT relation [46-50]. 
More generally, for $n$-point amplitude, under the usual gauge-fixing $z_{1}=\infty, z_{2}=1$, $z_{n}=0$, we wish to compute

$$
\mathcal{A}_{n}^{\mathrm{G}}=\sum_{s o l} \frac{z_{12}^{2} z_{2 n}^{2} z_{n 1}^{2}}{|\Phi|_{12 n}^{12 n}}\left(\mathrm{Pf}^{\prime} \Psi_{2 n \times 2 n}\right)\left(\mathrm{Pf}^{\prime} \widetilde{\Psi}_{2 n \times 2 n}\right)=\sum_{s o l} \frac{z_{12}^{2} z_{2 n}^{2} z_{n 1}^{2}}{|\Phi|_{12 n}^{12 n}}\left(\mathrm{Pf}^{\prime} \Psi_{2 n \times 2 n}\right)^{2} .
$$

In order to write down the reduced Pfaffian, we need to compute the Pfaffian of a $(2 n-2)$ $\times(2 n-2)$ matrix, which is quite complicated. Direct computation using the above formula is obviously very difficult, just like the direct computation of gravity amplitude by Feynman diagram. So we would like to follow the KLT formalism, and compute the gravity amplitude as square of Yang-Mills amplitudes.

An important property of the reduced Pfaffian is that, it can be expanded [3] as

$$
\operatorname{Pf}^{\prime} \Psi=\sum_{\alpha \in S_{n-3}} \frac{\sum_{\beta \in S_{n-3}} S[\alpha \mid \beta] A_{n}^{\mathrm{YM}}(1, \beta, n, n-1)}{\left(z_{1}-z_{\alpha_{2}}\right)\left(z_{\alpha_{2}}-z_{\alpha_{3}}\right) \cdots\left(z_{\alpha_{n-2}}-z_{n-1}\right)\left(z_{n-1}-z_{n}\right)\left(z_{n}-z_{1}\right)},
$$

where $\alpha, \beta$ are permutations of labels $2,3, \ldots, n-2$, and $S[\alpha \mid \beta]$ is the S-kernel. The appearance of $\mathcal{A}_{n}^{\mathrm{YM}}$ is a consequence of certain integrand summing over all $(n-3)$ ! solutions of scattering equations in the original derivation, and in the companion matrix method, it corresponds to the trace of that integrand when changing variables to companion matrices. In any event, it is a constant, and can be dragged out of the trace.

Using this expression, we can expand one $\mathrm{Pf}^{\prime} \Psi$ in the gravity amplitude,

$$
\begin{aligned}
\mathcal{A}_{n}^{\mathrm{G}} & =\sum_{\text {sol }} \sum_{\alpha \in S_{n-3}}\left(\frac{P\left(z_{3}, z_{4}, \ldots, z_{n-1}\right)}{Q\left(z_{3}, z_{4}, \ldots, z_{n-1}\right)}\right) \times \sum_{\beta \in S_{n-3}} S[\alpha \mid \beta] \mathcal{A}_{n}^{\mathrm{YM}}(1, \beta, n, n-1), \\
\bar{P} & \equiv \frac{z_{12}^{2} z_{2 n}^{2} z_{n 1}^{2}}{|\Phi|_{12 n}^{12 n}} \frac{\operatorname{Pf}^{\prime} \Psi_{2 n \times 2 n}}{z_{1 \alpha_{2}} z_{\alpha_{2} \alpha_{3}} \cdots z_{\alpha_{n-2}, n-1} z_{n-1, n} z_{n 1}}
\end{aligned}
$$

The trace $\operatorname{Tr}\left(P^{\prime}\left(T_{z_{i}}\right) Q^{\prime-1}\left(T_{z_{i}}\right)\right)$ for the set $\alpha$ gives $\mathcal{A}_{n}^{\mathrm{YM}}(1, \alpha, n-1, n)$, and the summation over permutations of $\alpha$ can be taken out of the trace, and we thereby arrive at the KLT relation. ${ }^{4}$

\subsection{Six-point amplitudes}

We proceed onto six-point amplitudes, i.e., $n=6$. Using the standard gauge-fixing $z_{1}=\infty$, $z_{2}=1, z_{6}=0$, Dolan-Goddard's polynomial form [37] of the scattering equations is given by

$$
\begin{aligned}
& f_{1}=s_{12}+s_{13} z_{3}+s_{14} z_{4}+s_{15} z_{5}, \\
& f_{2}=s_{123} z_{3}+s_{124} z_{4}+s_{125} z_{5}+s_{134} z_{3} z_{4}+s_{135} z_{3} z_{5}+s_{145} z_{4} z_{5}, \\
& f_{3}=s_{56} z_{3} z_{4}+s_{46} z_{3} z_{5}+s_{36} z_{4} z_{5}+s_{26} z_{3} z_{4} z_{5} .
\end{aligned}
$$

We can thus define the ideal $I=\left\langle f_{1}, f_{2}, f_{3}\right\rangle$ in the polynomial ring $\mathbb{C}\left[z_{3}, z_{4}, z_{5}\right]$. The degree of ideal $I$ is 6 , so according to Bézout's theorem, it has 6 solutions, though it is not possible to obtain analytic expressions for these solutions, as already seen in the 5-point cases. Let us then consider the companion matrix method.

\footnotetext{
${ }^{4}$ Note that the ordering of set $\alpha$ (or $\beta$ ) here defined in [3] is the reverse of that defined in [48].
} 
We generate the Gröbner basis for $I$ in Lexicographic ordering $z_{3} \prec z_{4} \prec z_{5}$. Analytically, the explicit expression of $\operatorname{GB}(I)$ is rather complicated, especially in the presence of so many parameters $s_{i j}$ in the ring. By varying the exponents to some high power, the polynomial reduction of the monomials $z_{3}^{a_{3}} z_{4}^{a_{4}} z_{5}^{a_{5}}$ (with $a_{i}$ from 0 to some finite number, say 20) over $G B(I)$ gives the monomial basis

$$
\mathrm{B}=\left\{1, z_{5}, z_{5}^{2}, z_{5}^{3}, z_{5}^{4}, z_{5}^{5}\right\}
$$

The polynomial reduction of $z_{3} \mathrm{~B}, z_{4} \mathrm{~B}$ and $z_{5} \mathrm{~B}$ over $\mathrm{GB}(I)$ gives the companion matrices $T_{z_{3}}, T_{z_{4}}, T_{z_{5}}$, which are $6 \times 6$ matrices. Again, we need to compute $P^{\prime}=$ $\left.P\right|_{z_{3} \rightarrow T_{z_{3}}, z_{4} \rightarrow T_{z_{4}}, z_{5} \rightarrow T_{z_{5}}} Q^{\prime}=\left.Q\right|_{z_{3} \rightarrow T_{z_{3}}, z_{4} \rightarrow T_{z_{4}}, z_{5} \rightarrow T_{z_{5}}}$, and the final amplitude is given by $\mathcal{A}_{6}=\operatorname{Tr}\left(P^{\prime} Q^{\prime-1}\right)$, without summing over all solutions of scattering equations.

Since the operations we need are multiplication of matrices, taking inverse or trace of matrices, so in principle it can be done analytically. However, the symbolic manipulation for $n=6$ case is quite complicated, especially when taking the inverse of matrix $Q^{\prime}$ and simplifying the tedious trace result in Mathematica, so we introduce random numeric kinematics - i.e., by Monte Carlo assignments of the parametres $s_{i j}$ — to get the final result. One will see that, as is customary with coefficient fields in polynomial rings, trying a few large prime numbers would suffice very quickly.

\subsubsection{Scalar $\phi^{3}$ theory}

We can write the amplitude as

$$
\begin{aligned}
\mathcal{A}_{6} & =\sum_{\text {sol }} \frac{z_{12}^{2} z_{26}^{2} z_{61}^{2}}{|\Phi|_{126}^{126}} \frac{1}{z_{12}^{2} z_{23}^{2} z_{34}^{2} z_{45}^{2} z_{56}^{2} z_{61}^{2}} \\
& =\sum_{\text {sol }} \frac{1}{|\Phi|_{126}^{126}\left(z_{3}-1\right)^{2}\left(z_{3}-z_{4}\right)^{2}\left(z_{4}-z_{5}\right)^{2} z_{5}^{2}} \equiv \sum_{z_{3}, z_{4}, z_{5} \in \text { sol }} \frac{P\left(z_{3}, z_{4}, z_{5}\right)}{Q\left(z_{3}, z_{4}, z_{5}\right)},
\end{aligned}
$$

where

$$
\Phi_{126}^{126}=\left(\begin{array}{lll}
\Phi_{33} & \Phi_{34} & \Phi_{35} \\
\Phi_{43} & \Phi_{44} & \Phi_{45} \\
\Phi_{53} & \Phi_{54} & \Phi_{55}
\end{array}\right), \quad|\Phi|_{126}^{126}=\operatorname{Det}\left(\Phi_{126}^{126}\right)
$$

Prime kinematic strategy. The idea is the following. Since we know for scalar $\phi^{3}$ theory, the final result of $\mathcal{A}_{6}$ should take the form

$$
\mathcal{A}_{6}=\sum_{1,1_{i}} \frac{c_{1}}{s_{11} s_{12} s_{13}}
$$

where $s_{1_{i}}$ are the independent Mandelstam variables of physical poles $s_{12}, s_{23}, s_{34}, s_{45}, s_{56}$, $s_{16}, s_{123}, s_{234}, s_{345}$, and the summation is over all possible products of three physical poles, e.g., $\frac{1}{s_{12} s_{23} s_{56}}, \frac{1}{s_{12} s_{45} s_{234}}$, etc. So in total we have $\left(\begin{array}{l}9 \\ 3\end{array}\right)=84$ terms, which we denote as $\mathcal{S}_{1}$, $1=1,2, \ldots 84$, and the amplitude is expanded as $\mathcal{A}_{6}=\sum_{1=1}^{84} c_{1} \mathcal{S}_{1}$, where $c_{1}$ is either 0 or 1 .

To each physical pole we now randomly assign a prime number, i.e., we are working with the much simpler polynomial ring $\mathbb{C}[z]$ instead of $\mathbb{C}(s)[z]$. In this case, the computation 
of $\operatorname{Tr}\left(P^{\prime} Q^{\prime-1}\right)$ is trivial within seconds, and the result as well as $\mathcal{S}_{1}$ 's are all numbers. Next, we shall find the solutions $\sum_{1=1}^{84} c_{1} \mathcal{S}_{1}=\operatorname{Tr}\left(P^{\prime} Q^{\prime-1}\right)$ for $c_{1}$ 's. However, doing this by bruteforce is impossible since there are $84 c_{1}$ 's and each one can take 0 or 1 , so one would go through all $2^{84}$ possibilities, which is far beyond any computational ability.

We therefore adopt the following strategy: instead of setting all coefficients to numbers, we can assign all physical poles to prime numbers except one pole. For example, we would leave $s_{345}$, to detect first the coefficients of $\mathcal{S}_{1}$ 's which contains the pole $\frac{1}{s_{345}}$. Keeping one symbolic variable $s_{345}$ would extend the computation time of $\operatorname{Tr}\left(P^{\prime} Q^{\prime-1}\right)$ up to minutes, but it is still very manageable, while keeping two or more symbolic variables would make the computation of $\operatorname{Tr}\left(P^{\prime} Q^{\prime-1}\right)$ in Mathematica very hard for a laptop.

Let us see the above strategy in action. Setting the kinematics (coefficient variables) as, e.g.,

$$
\begin{aligned}
& s_{12}=7, \quad s_{23}=37, \quad s_{34}=79, \quad s_{45}=97, \\
& s_{56}=131, \quad s_{16}=179, \quad s_{123}=181, \quad s_{234}=223 \text {, }
\end{aligned}
$$

while leaving $s_{345}$ free, we get

$$
\operatorname{Tr}\left(P^{\prime} Q^{\prime-1}\right)=-\frac{64909247478}{1878479042622679}-\frac{32736}{9601739 s_{345}} .
$$

Among the $\mathcal{S}_{1}$ 's, there are $\left(\begin{array}{l}8 \\ 2\end{array}\right)=28$ terms containing physical pole $s_{345}$, and the number marked by $\frac{1}{s_{345}}$ in $\operatorname{Tr}\left(P^{\prime} Q^{\prime-1}\right)$ should be expanded into these 28 terms. ${ }^{5}$ This is thus a problem in Egyptian fractions. By going through all $2^{28}$ possibilities of $c_{1}$, we find the unique expansion

$$
\frac{32736}{9601739} \frac{1}{s_{345}}=\left(\frac{1}{7 \times 79}+\frac{1}{79 \times 179}+\frac{1}{7 \times 97}+\frac{1}{97 \times 179}\right) \frac{1}{s_{345}},
$$

So mapping to the physical poles, we find that

$$
-\frac{1}{s_{12} s_{34} s_{345}}-\frac{1}{s_{16} s_{34} s_{345}}-\frac{1}{s_{12} s_{45} s_{345}}-\frac{1}{s_{16} s_{45} s_{345}}
$$

is a part of $\mathcal{A}_{6}$.

Now, we try to get more poles. Taking the kinematics as, e.g.,

$$
\begin{aligned}
& s_{12}=7 \text {, } \\
& s_{23}=37 \text {, } \\
& s_{34}=79 \text {, } \\
& s_{45}=97, \\
& s_{56}=131 \text {, } \\
& s_{16}=179 \text {, } \\
& s_{123}=181 \text {, } \\
& s_{345}=251 \text {, }
\end{aligned}
$$

while leaving $s_{234}$ free, we get

$$
\operatorname{Tr}\left(P^{\prime} Q^{\prime-1}\right)=-\frac{35960}{68541427 s_{234}}-\frac{13829207594}{302048840293589} .
$$

The part marked by the physical pole $s_{234}$ can be uniquely expanded as

$$
\frac{35960}{68541427} \frac{1}{s_{234}}=\left(\frac{1}{37 \times 179}+\frac{1}{79 \times 179}+\frac{1}{37 \times 131}+\frac{1}{79 \times 131}\right) \frac{1}{s_{234}},
$$

\footnotetext{
${ }^{5}$ In fact, using the compatibility among poles, we can greatly reduce the number of possible combinations of poles. We will consider this fact in latter examples.
} 
thus

$$
-\frac{1}{s_{16} s_{23} s_{234}}-\frac{1}{s_{16} s_{34} s_{234}}-\frac{1}{s_{23} s_{56} s_{234}}-\frac{1}{s_{34} s_{56} s_{234}}
$$

is also a part of $\mathcal{A}_{6}$. With the same procedure, we find that for physical pole $s_{123}$,

$$
-\frac{1}{s_{12} s_{45} s_{123}}-\frac{1}{s_{23} s_{45} s_{123}}-\frac{1}{s_{12} s_{56} s_{123}}-\frac{1}{s_{23} s_{56} s_{123}}
$$

is also part of $\mathcal{A}_{6}$. Finally, we need to determine the coefficients $c_{1}$ of $\mathcal{S}_{1}$ 's without physical poles $s_{123}, s_{234}, s_{345}$. There are in total $\left(\begin{array}{l}6 \\ 3\end{array}\right)=20$ terms. Taking the kinematics as, e.g.,

$$
\begin{aligned}
& s_{12}=7, \quad s_{23}=37, \quad s_{34}=79, \quad s_{45}=97, \\
& s_{56}=131, \quad s_{16}=179, \quad s_{123}=181, \quad s_{234}=223, \quad s_{345}=251 \text {, }
\end{aligned}
$$

computing the $\operatorname{Tr}\left(P^{\prime} Q^{\prime-1}\right)$ and extracting the contributions from results $(4.69),(4.72),(4.73)$, the remaining result can be uniquely expanded as

$$
-\frac{714874}{46539628933}=-\frac{1}{7 \times 79 \times 131}-\frac{1}{37 \times 97 \times 179},
$$

so the last part for $\mathcal{A}_{6}$ is

$$
-\frac{1}{s_{12} s_{34} s_{56}}-\frac{1}{s_{16} s_{23} s_{45}}
$$

Putting all the above together, we therefore conclude that

$$
\begin{aligned}
\mathcal{A}_{6}= & -\left(\frac{1}{s_{12} s_{34} s_{56}}+\frac{1}{s_{16} s_{23} s_{45}}+\frac{1}{s_{12} s_{45} s_{123}}+\frac{1}{s_{23} s_{45} s_{123}}+\frac{1}{s_{12} s_{56} s_{123}}\right. \\
& +\frac{1}{s_{23} s_{56} s_{123}}+\frac{1}{s_{12} s_{34} s_{345}}+\frac{1}{s_{16} s_{34} s_{345}}+\frac{1}{s_{12} s_{45} s_{345}}+\frac{1}{s_{16} s_{45} s_{345}} \\
& \left.+\frac{1}{s_{16} s_{23} s_{234}}+\frac{1}{s_{16} s_{34} s_{234}}+\frac{1}{s_{23} s_{56} s_{234}}+\frac{1}{s_{34} s_{56} s_{234}}\right) .
\end{aligned}
$$

This prime-numeric method can be applied to all the cases of $n=6$ amplitudes of scalar $\phi^{3}$ theory.

\subsubsection{Yang-Mills theory}

For Yang-Mills theory, when $n=6$, we meet the first "not so simple" gluon amplitude, i.e., the next-MHV amplitude, so it is worthwhile to verify the companion matrix method with this non-trivial example. To illustrate, let us consider the split helicity amplitude $\mathcal{A}_{6}^{\mathrm{YM}}\left(g_{1}^{-}, g_{2}^{-}, g_{3}^{-}, g_{4}^{+}, g_{5}^{+}, g_{6}^{+}\right)$, and choose the reference momenta as $r_{1}=r_{2}=r_{3}=k_{4}$, $r_{4}=r_{5}=r_{6}=k_{3}$, so that only $\widetilde{\epsilon}_{1,5}, \widetilde{\epsilon}_{1,6}, \widetilde{\epsilon}_{2,5}, \widetilde{\epsilon}_{2,6}$ are non-zero. The object we want to compute is

$$
\mathcal{A}_{6}^{\mathrm{YM}}=\sum_{\text {sol }} \frac{z_{12}^{2} z_{26}^{2} z_{61}^{2}}{|\Phi|_{126}^{126}} \frac{\mathrm{Pf}^{\prime} \Psi_{12 \times 12}}{z_{12} z_{23} z_{34} z_{45} z_{56} z_{61}} \equiv \sum_{z_{3}, z_{4}, z_{5} \in \text { sol }} \frac{P^{\mathrm{YM}}\left(z_{3}, z_{4}, z_{5}\right)}{Q^{\mathrm{YM}}\left(z_{3}, z_{4}, z_{5}\right)} .
$$


Here both the Jacobian $|\Phi|_{126}^{126}$ and reduced Pfaffian $\operatorname{Pf}^{\prime} \Psi_{12 \times 12}$ are very complicated, so it is almost impossible to compute it analytically. As in the scalar $\phi^{3}$ example, we can follow the semi-analytic procedure, and set the physical poles as some prime numbers, while keeping $\Psi_{12 \times 12}\left(\right.$ all $\widetilde{\epsilon}_{i, j}=\epsilon_{i} \epsilon_{j}, \kappa_{i, j}=\epsilon_{i} k_{j}$ and $k_{i} k_{j}$ in $\left.\Psi\right)$ analytic. In this case, the ideal and Gröbner basis are just algebraic systems of polynomials with integer coefficients, while the elements of companion matrices are rational numbers. So the computation is very fast.

The Jacobian under the chosen gauge-fixing is

$$
\frac{1}{|\Phi|_{126}^{126}}=\frac{z_{3}^{2} z_{4}^{2} z_{5}^{2}\left(z_{3}-1\right)^{2}\left(z_{4}-1\right)^{2}\left(z_{5}-1\right)^{2}\left(z_{3}-z_{4}\right)^{2}\left(z_{3}-z_{5}\right)^{2}\left(z_{4}-z_{5}\right)^{2}}{D_{\Phi}\left(z_{3}, z_{4}, z_{5}\right)},
$$

where $D_{\Phi}$ is polynomial in $z_{3}, z_{4}, z_{5}$. The reduced Pfaffian together with the factor of scalar part give

$$
\frac{N_{\Psi}\left(z_{3}, z_{4}, z_{5}, \widetilde{\epsilon}_{i, j}, \kappa_{i, j}, k_{i} k_{j}\right)}{z_{3} z_{4} z_{5}^{2}\left(z_{3}-1\right)^{2}\left(z_{4}-1\right)\left(z_{5}-1\right)\left(z_{3}-z_{4}\right)^{2}\left(z_{3}-z_{5}\right)\left(z_{4}-z_{5}\right)^{2}}
$$

under the chosen gauge-fixing for some polynomial numerator $N_{\Phi}$. So we have

$$
P^{\mathrm{YM}}\left(z_{3}, z_{4}, z_{5}\right)=z_{3} z_{4}\left(z_{4}-1\right)\left(z_{5}-1\right)\left(z_{3}-z_{5}\right) N_{\Psi}, \quad Q^{\mathrm{YM}}\left(z_{3}, z_{4}, z_{5}\right)=D_{\Phi} .
$$

Note that $N_{\Psi}$ originates from the Pfaffian of a $10 \times 10$ antisymmetric matrix, where by definition, each term in the Pfaffian is a product of five elements in the matrix. So each term in $N_{\Psi}$ is a product of five elements selected from $\widetilde{\epsilon}_{i, j}, \kappa_{i, j}, k_{i} k_{j}$, combined with a monomial of $z_{3}, z_{4}, z_{5}$, for example, $2 z_{3}^{3} z_{4}^{4} \widetilde{\epsilon}_{2,6} \kappa_{1,2} \kappa_{3,1} \kappa_{4,1} \kappa_{5,1}$.

Finally we can take the replacement $P^{\prime \mathrm{YM}}=\left.P^{\mathrm{YM}}\right|_{z_{i} \rightarrow T_{z_{i}}}, Q^{\prime \mathrm{YM}}=\left.Q^{\mathrm{YM}}\right|_{z_{i} \rightarrow T_{z_{i}}}$ and compute the trace $\operatorname{Tr}\left(P^{\prime \mathrm{YM}}\left(Q^{\prime \mathrm{YM}}\right)^{-1}\right)$. It is given as

$$
\begin{aligned}
\operatorname{Tr}\left(P^{\prime \mathrm{YM}}\left(Q^{\prime \mathrm{YM}}\right)^{-1}\right)= & \frac{44}{6141149} \widetilde{\epsilon}_{2,6} \kappa_{1,2} \kappa_{3,2} \kappa_{4,1} \kappa_{5,1}-\frac{1}{877307} \widetilde{\epsilon}_{2,5} \kappa_{1,3} \kappa_{3,2} \kappa_{4,1} \kappa_{6,2} \\
& +500 \text { more terms } .
\end{aligned}
$$

Using the techniques shown in scalar $\phi^{3}$ theory, we can uniquely decompose the rational numbers as

$$
\frac{44}{6141149}=\frac{1}{7 \times 181 \times 131}+\frac{1}{181 \times 37 \times 131}, \quad \frac{1}{877307}=\frac{1}{181 \times 37 \times 131},
$$

so we can conclude that

$$
\begin{aligned}
\operatorname{Tr}\left(P^{\prime \mathrm{YM}}\left(Q^{\prime \mathrm{YM}}\right)^{-1}\right)= & \left(\frac{1}{s_{12} s_{123} s_{56}}+\frac{1}{s_{123} s_{23} s_{56}}\right) \widetilde{\epsilon}_{2,6} \kappa_{1,2} \kappa_{3,2} \kappa_{4,1} \kappa_{5,1} \\
& -\frac{1}{s_{123} s_{23} s_{56}} \widetilde{\epsilon}_{2,5} \kappa_{1,3} \kappa_{3,2} \kappa_{4,1} \kappa_{6,2}+500 \text { more terms } .
\end{aligned}
$$

Rewriting them as spinor products and applying the simplification algorithm for spinor expression, we get a one-page long result, which remarkably agrees with the known answers $[54,55]$. 


\subsection{Seven-point amplitudes}

The companion matrices $T_{z_{i}}$ are simultaneously diagonalizable, and according to Stickelberger's theorem, the complex roots $z_{i}$ of ideal $I$ are the vectors of simultaneous eigenvalues of the companion matrices $T_{z_{i}}$. Thus when they are evaluated in the diagonal form, the matrices $P^{\prime}=\left.P\right|_{z_{i} \rightarrow T_{z_{i}}}, Q^{\prime}=\left.Q\right|_{z_{i} \rightarrow T_{z_{i}}}, P^{\prime} Q^{\prime-1}$ are also diagonal, and it builds the oneto-one mapping between diagonal elements of $(n-3) ! \times(n-3)$ ! matrix $P^{\prime} Q^{\prime-1}$ and the integrand $P / Q$ evaluated at the $(n-3)$ ! complex solutions of scattering equations. To demonstrate this, let us go through a 7-point example of scalar $\phi^{3}$ theory.

As usual, let us gauge fixing $z_{1}=\infty, z_{2}=1, z_{7}=0$, and the amplitude is given by

$$
\mathcal{A}_{7}=\sum_{\text {sol }} \frac{z_{12}^{2} z_{27}^{2} z_{71}^{2}}{|\Phi|_{127}^{127}} \frac{1}{z_{12}^{2} z_{23}^{2} z_{34}^{2} z_{45}^{2} z_{56}^{2} z_{67}^{2} z_{71}^{2}} \equiv \sum_{z_{3}, z_{4}, z_{5}, z_{6} \in \text { sol }} \frac{P\left(z_{3}, z_{4}, z_{5}, z_{6}\right)}{Q\left(z_{3}, z_{4}, z_{5}, z_{6}\right)}
$$

where

$$
\Phi_{127}^{127}=\left(\begin{array}{llll}
\Phi_{33} & \Phi_{34} & \Phi_{35} & \Phi_{36} \\
\Phi_{43} & \Phi_{44} & \Phi_{45} & \Phi_{46} \\
\Phi_{53} & \Phi_{54} & \Phi_{55} & \Phi_{56} \\
\Phi_{63} & \Phi_{64} & \Phi_{65} & \Phi_{66}
\end{array}\right), \quad|\Phi|_{127}^{127}=\operatorname{Det}\left(\Phi_{127}^{127}\right)
$$

The Dolan-Goddard polynomial form [37] of the scattering equations is given by

$$
\begin{aligned}
f_{1}= & s_{12}+s_{13} z_{3}+s_{14} z_{4}+s_{15} z_{5}+s_{16} z_{6}, \\
f_{2}= & s_{123} z_{3}+s_{124} z_{4}+s_{125} z_{5}+s_{126} z_{6} \\
& +s_{134} z_{3} z_{4}+s_{135} z_{3} z_{5}+s_{136} z_{3} z_{6}+s_{145} z_{4} z_{5}+s_{146} z_{4} z_{6}+s_{156} z_{5} z_{6}, \\
f_{3}= & s_{1234} z_{3} z_{4}+s_{1235} z_{3} z_{5}+s_{1236} z_{3} z_{6}+s_{1245} z_{4} z_{5}+s_{1246} z_{4} z_{6}+s_{1256} z_{5} z_{6} \\
& +s_{1345} z_{3} z_{4} z_{5}+s_{1346} z_{3} z_{4} z_{6}+s_{1356} z_{3} z_{5} z_{6}+s_{1456} z_{4} z_{5} z_{6}, \\
f_{4}= & s_{67} z_{3} z_{4} z_{5}+s_{57} z_{3} z_{4} z_{6}+s_{47} z_{3} z_{5} z_{6}+s_{37} z_{4} z_{5} z_{6}+s_{27} z_{3} z_{4} z_{5} z_{6} .
\end{aligned}
$$

We can define the ideal $I=\left\langle f_{1}, f_{2}, f_{3}, f_{4}\right\rangle$ in polynomial ring $\mathbb{C}\left[z_{3}, z_{4}, z_{5}, z_{6}\right]$, and generate the Gröbner basis of $I$ in Lexicographic order $z_{3} \prec z_{4} \prec z_{5} \prec z_{6}$. The degree of ideal $I$ is 24 , so the variety of ideal $I$ is given by 24 point solutions for which there are no closed form solutions.

Let us set the kinematics (all physical poles) as some prime numbers,

$$
\begin{aligned}
& s_{12}=5, \quad s_{23}=37, \quad s_{34}=43, \quad s_{45}=61, \quad s_{56}=97, \quad s_{67}=101, \quad s_{17}=139, \\
& s_{123}=151, \quad s_{234}=163, \quad s_{345}=191, \quad s_{456}=211, \quad s_{567}=223, \quad s_{671}=251, \quad s_{712}=263
\end{aligned}
$$

in the following computation. The solutions of scattering equations $f_{i}=0, i=1,2,3,4$ requires computing the roots of equations of degree 24 , which has no closed form in radicals. Doing it numerically, we get 24 solutions 
sol $_{1}: z_{3}=20.9071, z_{4}=1.66835, z_{5}=7.08198, z_{6}=-64.2332$,

$\operatorname{sol}_{2}: z_{3}=1.4223-0.318993 \dot{\mathrm{i}}, z_{4}=12.204-5.48743 \dot{\mathrm{i}}, \quad z_{5}=0.342956-0.477119 \dot{\mathrm{i}}, z_{6}=51.9097-32.886 \dot{\mathrm{i}}$,

$\operatorname{sol}_{3}: z_{3}=1.4223+0.318993 \dot{\mathbf{i}}, z_{4}=12.204+5.48743 \dot{\mathbf{i}}, \quad z_{5}=0.342956+0.477119 \dot{\mathbf{i}}, z_{6}=51.9097+32.886 \dot{\mathbf{i}}$,

sol $_{4}: z_{3}=27.2316, z_{4}=1.76178, z_{5}=13.0497, z_{6}=-12.5157$,

sol $_{5}: z_{3}=1.34598, z_{4}=-3.76733, z_{5}=-1.28282, z_{6}=-56.7763$,

$\operatorname{sol}_{6}: z_{3}=4.92534+1.82303 \dot{\mathrm{i}}, z_{4}=2.04236+0.47052 \dot{\mathrm{i}}, z_{5}=0.12331+0.73366 \dot{\mathrm{i}}, \quad z_{6}=-36.88-1.74857 \dot{\mathbf{i}}$,

$\operatorname{sol}_{7}: z_{3}=4.92534-1.82303 \dot{\mathrm{i}}, z_{4}=2.04236-0.47052 \dot{\mathrm{i}}, z_{5}=0.12331-0.73366 \dot{\mathrm{i}}, z_{6}=-36.88+1.74857 \dot{\mathrm{i}}$,

sol $_{8}: z_{3}=-11.2804, z_{4}=3.5116, z_{5}=-6.80042, z_{6}=-1.26394$,

sol $_{9}: z_{3}=1.19261, z_{4}=-8.20104, z_{5}=3.07784, z_{6}=6.22689$,

sol $_{10}: z_{3}=1.18325+1.93745 \dot{\mathrm{i}}, \quad z_{4}=0.29585-0.48639 \dot{\mathrm{i}}, \quad z_{5}=0.56997+1.11008 \dot{\mathrm{i}}, \quad z_{6}=0.15405-0.39359 \dot{\mathrm{i}}$,

$\operatorname{sol}_{11}: z_{3}=1.18325-1.93745 \dot{\mathrm{i}}, z_{4}=0.29585+0.48639 \dot{\mathrm{i}}, z_{5}=0.56997-1.11008 \dot{\mathrm{i}}, z_{6}=0.15405+0.39359 \dot{\mathrm{i}}$,

sol $_{12}: z_{3}=-4.76521, z_{4}=-3.05026, z_{5}=-1.6908, z_{6}=-0.488528$,

$\operatorname{sol}_{13}: z_{3}=0.576445, z_{4}=-3.05135, z_{5}=1.14498, z_{6}=0.712806$,

$\operatorname{sol}_{14}: z_{3}=1.78095+0.41639 \dot{\mathrm{i}}, z_{4}=2.1103-0.60663 \dot{\mathrm{i}}, z_{5}=0.52752+0.29927 \dot{\mathrm{i}}, z_{6}=2.3283-1.39061 \dot{\mathrm{i}}$,

$\operatorname{sol}_{15}: z_{3}=1.78095-0.41639 \dot{\mathrm{i}}, z_{4}=2.1103+0.60663 \dot{\mathrm{i}}, z_{5}=0.52752-0.29927 \dot{\mathrm{i}}, \quad z_{6}=2.3283+1.39061 \dot{\mathrm{i}}$,

sol $_{16}: z_{3}=1.86192, z_{4}=0.877999, z_{5}=0.795994, z_{6}=0.601979$,

$\operatorname{sol}_{17}: z_{3}=1.65547, z_{4}=1.9848, z_{5}=0.493798, z_{6}=2.31186$,

sol $_{18}: z_{3}=0.327576, z_{4}=-0.0855936, z_{5}=0.212916, z_{6}=-0.0559545$,

$\operatorname{sol}_{19}: z_{3}=0.307828, z_{4}=0.645287, z_{5}=0.0420044, z_{6}=0.46483$

sol $_{20}: z_{3}=0.174313, z_{4}=0.120642, z_{5}=0.0855984, z_{6}=0.0445606$,

sol $_{21}: z_{3}=0.031819, z_{4}=0.15022, z_{5}=0.00455376, z_{6}=0.0545382$,

sol $_{22}: z_{3}=0.0191033, z_{4}=0.0765079, z_{5}=0.0145344, z_{6}=0.00921803$,

sol $_{23}: z_{3}=-0.100486, z_{4}=-0.0950558, z_{5}=-0.00857861, z_{6}=-0.0892275$,

sol $_{24}: z_{3}=-0.0162083, z_{4}=0.0167369, z_{5}=0.00970032, z_{6}=-0.0265855$,

and the integrand summing over all solutions is given by

$$
\sum_{\text {sol }_{i}, i=1}^{24} \frac{P\left(z_{3}, z_{4}, z_{5}, z_{6}\right)}{Q\left(z_{3}, z_{4}, z_{5}, z_{6}\right)}=1.99605 \times 10^{-6} .
$$

Let us now turn to the companion matrix method. The monomial basis over $\operatorname{GB}(I)$ is given by 24 elements

$$
\begin{aligned}
\mathrm{B}= & \left\{1, z_{6}, z_{6}^{2}, z_{6}^{3}, z_{6}^{4}, z_{6}^{5}, z_{6}^{6}, z_{6}^{7}, z_{6}^{8}, z_{6}^{9}, z_{6}^{10}, z_{6}^{11}, z_{6}^{12},\right. \\
& \left.z_{6}^{13}, z_{6}^{14}, z_{6}^{15}, z_{6}^{16}, z_{6}^{17}, z_{6}^{18}, z_{6}^{19}, z_{6}^{20}, z_{6}^{21}, z_{6}^{22}, z_{6}^{23}\right\} .
\end{aligned}
$$

Accordingly, by polynomial reduction of $z_{i} \mathrm{~B}, i=3,4,5,6$ over $\mathrm{GB}(I)$, we can get the companion matrices $T_{z_{i}}, i=3,4,5,6$, which are $24 \times 24$ matrix and satisfying $T_{z_{i}} \mathrm{~B}=z_{i} \mathrm{~B}$. In order to compute $\mathcal{A}_{7}$, we can proceed as usual by computing $\operatorname{Tr}\left(\left.\left.P\right|_{z_{i} \rightarrow T_{z_{i}}} Q\right|_{z_{i} \rightarrow T_{z_{i}}} ^{-1}\right)$, and the result is

$$
\operatorname{Tr}\left(P^{\prime} Q^{\prime-1}\right)=\frac{19260317055974762778118}{9649229470008137021319652355} \approx 1.99605 \times 10^{-6},
$$


which agrees with the numeric result given by summing over all solutions of scattering equations. Again we see that, since the computation only involves basic manipulations on matrix, we are able to get the closed form result, and show that the final result is rational functions of Mandelstam variables.

The companion matrices are simultaneously diagonalizable. We can choose $T_{z_{6}}$ and compute its eigenvectors, since $T_{z_{6}}$ is the simplest companion matrix by definition. Such computation involves finding the roots of equations of degree 24, which prohibits analytic solution. Now, $T_{z_{6}}$ has 24 column eigenvectors $u_{i}, i=1, \ldots, 24$, and from them we can define the transformation matrix $U=\left(u_{1}, \ldots, u_{24}\right)_{24 \times 24}$. Then $T_{z_{i}}^{d}=U^{-1} T_{z_{i}} U, i=3,4,5,6$ are all diagonal matrices, explicitly given as

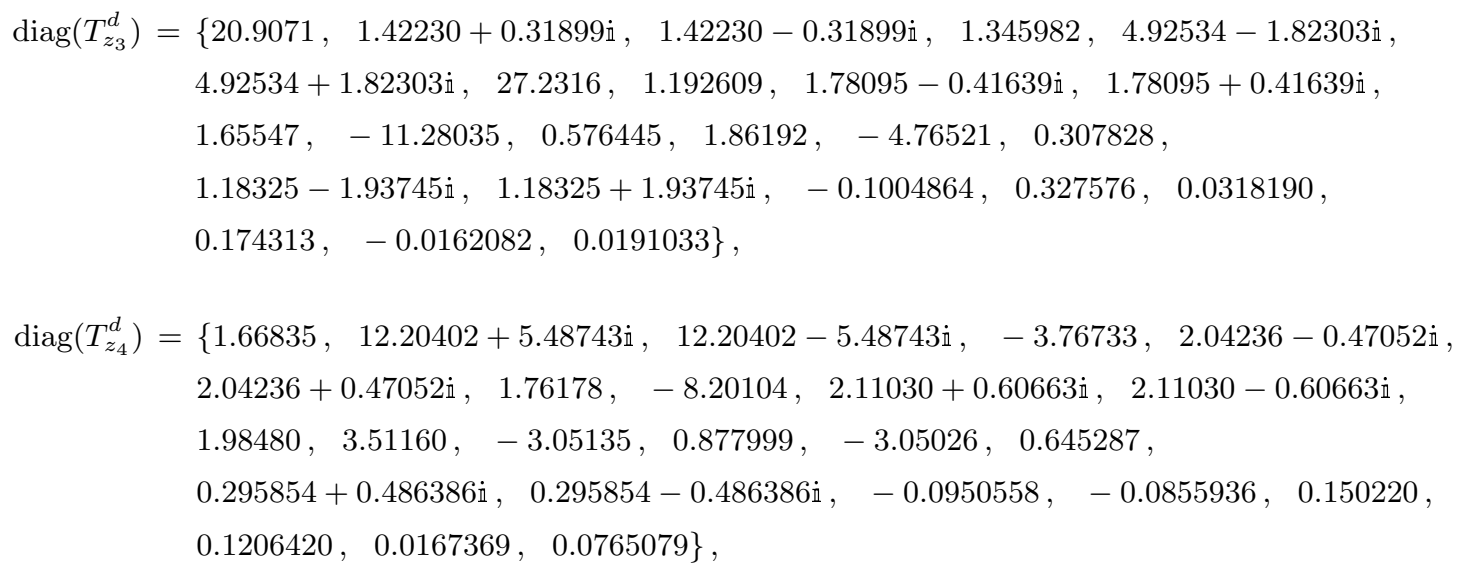

and

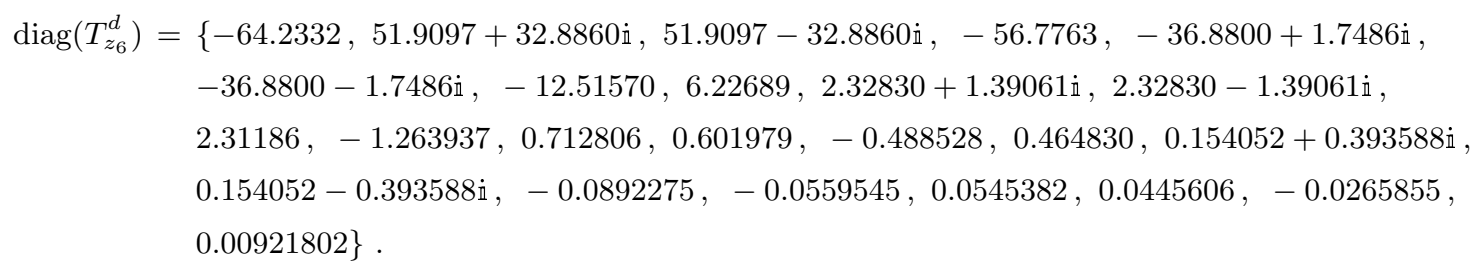

It can be checked directly that, each set of diagonal elements $\left\{\left(T_{z_{3}}\right)_{i, i}, \quad\left(T_{z_{4}}\right)_{i, i}\right.$, $\left.\left(T_{z_{5}}\right)_{i, i},\left(T_{z_{6}}\right)_{i, i}\right\}$ corresponds to a set of solution $\left\{z_{3}^{\text {sol }_{j}}, z_{4}^{\text {sol }_{j}}, z_{5}^{\text {sol }_{j}}, z_{6}^{\text {sol }_{j}}\right\}$ of scattering equations. Thus each diagonal element of matrix $P^{\prime}\left(T_{z_{i}}^{d}\right) Q^{\prime-1}\left(T_{z_{i}}^{d}\right)$ is identical to the integrand $P / Q$ evaluated at one solution of scattering equations, and the equivalence between results of these two methods is obvious.

With the arithmetic result, it is possible to determine the terms appearing in amplitude by setting appropriate kinematics. In fact, in this example, we know that the result should 
be the sum

$$
\mathcal{A}_{7}=\sum_{1,1_{i}} \frac{c_{1}}{s_{11} s_{12} s_{13} s_{14}}
$$

where naively the summation is over all possible products of 4 physical poles $s_{1}$, i.e., $\left(\begin{array}{c}14 \\ 4\end{array}\right)=1001$ terms with $c_{1}, 1=1, \ldots 1001$, being either zero or one. By choosing 1001 different group of kinematics for physical poles, we get 1001 linear equations of (4.94), and solving them gives the $c_{1}$.

Indeed, the number of terms grows very fast with $n$ in (4.94). The number of independent poles is $n^{\prime}=\frac{(n-1)(n-2)}{2^{2}}-1$ for massless theory, while the number of possible terms in the expansion is $\left(\begin{array}{c}n^{\prime} \\ n-3\end{array}\right)$. For $n=8$, the number is 15504 , and for $n=9$ the number is 296010. So it is not very doable when $n$ is large. However, for $\phi^{3}$ theory, the number of color-ordered diagram is much smaller, and the counting is $\frac{2^{n-2}(2 n-5) ! !}{(n-1) !}$. So for $n=7$, the possible terms appearing in (4.94) is 42 (an auspicious number). For $n=8$, the number is 132 , and for $n=9$, the number is 429 , etc. If we restrict to the 42 possible terms in (4.94), then it is possible to compute the coefficients $c_{1}$ by choosing one set of kinematics.

One can let each physical pole be assigned a random prime number, and compute $\operatorname{Tr}\left(P^{\prime} Q^{\prime-1}\right)$ and then let Mathematica go through all $2^{42}$ possibilities of $c_{1}$ 's to find the summation $\sum_{1=1}^{42} \frac{c_{1}}{s_{11} s_{12} s_{1_{3}} s_{1_{4}}}=\operatorname{Tr}\left(P^{\prime} Q^{\prime-1}\right)$. If the prime numbers in kinematic variables are distributed randomly in a very large scale, e.g., primes between 2 to 10000, then usually we can find one unique solution for $c_{1}$ in the spirit of Egyptian fractions. This enables us to do one computation and fix all coefficients.

For example, let us compute

$$
\mathcal{A}_{7}^{\prime}=\sum_{\text {sol }} \frac{z_{12}^{2} z_{27}^{2} z_{71}^{2}}{|\Phi|_{127}^{127}} \frac{1}{z_{12} z_{23} z_{34} z_{45} z_{56} z_{67} z_{71} z_{12} z_{24} z_{45} z_{57} z_{76} z_{63} z_{31}} .
$$

With the kinematics shown in (4.90), we find the unique decomposition

$$
\operatorname{Tr}\left(P^{\prime} Q^{\prime-1}\right)=\frac{284}{1037296765}=\frac{1}{5 \times 61 \times 101 \times 151}+\frac{1}{5 \times 101 \times 151 \times 233},
$$

which indicates that

$$
A_{7}^{\prime}=\frac{1}{s_{12} s_{45} s_{67} s_{123}}+\frac{1}{s_{12} s_{67} s_{123} s_{567}},
$$

and agrees with the result given by CHY mapping rules [30, 31].

There is a way to directly determine whether a certain term $\frac{1}{s_{11} s_{12} s_{13} s_{14}}$ is present in the result or not, by setting the kinematics $s_{11}=a, s_{12}=a^{2}, s_{13}=a^{4}, s_{14}=a^{8}$, and others random primes not equaling to $a$. If this term exists, then the denominator has a factor $a^{15}$. Again in the $\mathcal{A}_{7}^{\prime}$ example, if we instead set $s_{12}=5, s_{45}=5^{2}, s_{67}=5^{4}, s_{123}=5^{8}$, then the result is $\frac{248}{5^{15} \times 223}$, thus $\frac{1}{s_{1_{1}} s_{1_{2}} s_{13} s_{14}}$ is a term in $A_{7}^{\prime}$. However, if we set $s_{12}=5, s_{56}=5^{2}$, $s_{67}=5^{4}, s_{123}=5^{8}$, then the result is $\frac{284}{5^{13} \times 61 \times 223}$. This indicates that $\frac{1}{s_{12} s_{56} s_{67} s_{123}}$ is not a term in $\mathcal{A}_{7}^{\prime}$, while the $5^{13}$ factor indicates that possible terms involving $\frac{1}{s_{12} s_{67} s_{123}}$ must exist, which provides further information for detecting other existing terms. By this way, we can check all possible terms by setting kinematics for each one. 
The number of solutions for scattering equations grows as $(n-3)$ !, while the companion matrix grows as $(n-3) ! \times(n-3)$ !. When $n=8$, we need to invert the matrix $Q_{120 \times 120}^{\prime}$, and at $n=9$, the matrix $Q_{720 \times 720}^{\prime}$, etc. This sets the limitation on the computation of higher $n$.

\section{Conclusions and outlook}

In this paper, motivated by the explanation of equivalence of different integrands in the CHY setup, we propose a new method using companion matrices, borrowed from the study of zero-dimensional ideals in computational algebraic geometry, to evaluate the integrand. One advantage of the method is that the rationality of final integral is obvious. Thus although our method may not be as efficient as the one proposed in [30,31,36], it does give a new angle to study the important problem of scattering amplitudes.

As shown in the plethora of examples, when the number of external legs grows, the analytic expression of companion matrix becomes harder. In fact, when $n \geq 6$, the best way to do it is by assigning the kinematic variables to random prime numbers in order to reconstruct the analytic result. The salient feature of our method is that it is purely linear-algebraic, involving nothing more than finding the inverse and trace of matrices. The linearity of the trace, for example, was demonstrated to immediately lead to non-trivial identities in the amplitudes.

Now, since the physical problem is very symmetric as can be seen by the polynomials given in (2.15) and (2.16), one is confronted with an immediate mathematical challenge. If we could analytically find, say by induction, the Gröbner basis and subsequent monomial basis for the polynomial form of the scattering equations in some appropriate lexicographic ordering, then one would find a recursive way to construct the companion matrix explicitly, much like the recursive construction of tree-level amplitude by using BCFW deformation $[16,17]$. Working out this construction is hard but worthwhile, as it would give explicit analytic results for the amplitudes and provide a deeper understanding of the CHY formalism.

\section{Acknowledgments}

We would like to thank Yang Zhang for discussion and sharing the similar idea of using computational algebraic geometry. We would also like to thank C. Baadsgaard, N. BjerrumBohr, P. H. Damgaard for the discussion. RH acknowledges discussions with Qingjun Jin. BF is grateful to the Qiu-Shi Fund and the Chinese NSF under contracts No.11031005, No.11135006 and No.11125523; he would like to thank the hospitality of the Niels Bohr International Academy and City University, London. YHH would like to thank the Science and Technology Facilities Council, U.K., for grant ST/J00037X/1, the Chinese Ministry of Education, for a Chang-Jiang Chair Professorship at NanKai University as well as the City of Tian-Jin for a Qian-Ren Scholarship, as well as City University, London and Merton College, Oxford, for their enduring support. RH would also like to thank the supporting from Chinese Postdoctoral Administrative Committee. 
Open Access. This article is distributed under the terms of the Creative Commons Attribution License (CC-BY 4.0), which permits any use, distribution and reproduction in any medium, provided the original author(s) and source are credited.

\section{References}

[1] F. Cachazo, S. He and E.Y. Yuan, Scattering equations and Kawai-Lewellen-Tye orthogonality, Phys. Rev. D 90 (2014) 065001 [arXiv:1306.6575] [InSPIRE].

[2] F. Cachazo, S. He and E.Y. Yuan, Scattering of Massless Particles in Arbitrary Dimensions, Phys. Rev. Lett. 113 (2014) 171601 [arXiv:1307.2199] [INSPIRE].

[3] F. Cachazo, S. He and E.Y. Yuan, Scattering of Massless Particles: Scalars, Gluons and Gravitons, JHEP 07 (2014) 033 [arXiv:1309.0885] [INSPIRE].

[4] F. Cachazo, S. He and E.Y. Yuan, Einstein-Yang-Mills Scattering Amplitudes From Scattering Equations, JHEP 01 (2015) 121 [arXiv:1409.8256] [INSPIRE].

[5] F. Cachazo, S. He and E.Y. Yuan, Scattering Equations and Matrices: From Einstein To Yang-Mills, DBI and NLSM, JHEP 07 (2015) 149 [arXiv: 1412.3479] [INSPIRE].

[6] D. Fairlie and D. Roberts, Dual Models without Tachyons - a New Approach, unpublished, Durham preprint PRINT-72-2440 (1972).

[7] D. Roberts, Mathematical Structure of Dual Amplitudes, Ph.D. Thesis, Durham University, Durham U.K. (1972).

[8] D.B. Fairlie, A Coding of Real Null Four-Momenta into World-Sheet Co-ordinates, Adv. Math. Phys. 2009 (2009) 284689 [arXiv:0805.2263] [INSPIRE].

[9] D.J. Gross and P.F. Mende, String Theory Beyond the Planck Scale, Nucl. Phys. B 303 (1988) 407 [INSPIRE].

[10] E. Witten, Parity invariance for strings in twistor space, Adv. Theor. Math. Phys. 8 (2004) 779 [hep-th/0403199] [INSPIRE].

[11] P. Caputa and S. Hirano, Observations on Open and Closed String Scattering Amplitudes at High Energies, JHEP 02 (2012) 111 [arXiv:1108.2381] [INSPIRE].

[12] P. Caputa, Lightlike contours with fermions, Phys. Lett. B 716 (2012) 475 [arXiv:1205.6369] [INSPIRE].

[13] Y. Makeenko and P. Olesen, The QCD scattering amplitude from area behaved Wilson loops, Phys. Lett. B 709 (2012) 285 [arXiv:1111.5606] [INSPIRE].

[14] F. Cachazo, Fundamental BCJ Relation in $N=4$ SYM From The Connected Formulation, arXiv: 1206.5970 [INSPIRE].

[15] L. Dolan and P. Goddard, Proof of the Formula of Cachazo, He and Yuan for Yang-Mills Tree Amplitudes in Arbitrary Dimension, JHEP 05 (2014) 010 [arXiv:1311.5200] [INSPIRE].

[16] R. Britto, F. Cachazo and B. Feng, New recursion relations for tree amplitudes of gluons, Nucl. Phys. B 715 (2005) 499 [hep-th/0412308] [INSPIRE].

[17] R. Britto, F. Cachazo, B. Feng and E. Witten, Direct proof of tree-level recursion relation in Yang-Mills theory, Phys. Rev. Lett. 94 (2005) 181602 [hep-th/0501052] [INSPIRE].

[18] L. Mason and D. Skinner, Ambitwistor strings and the scattering equations, JHEP 07 (2014) 048 [arXiv: 1311.2564] [INSPIRE]. 
[19] N. Berkovits, Infinite Tension Limit of the Pure Spinor Superstring, JHEP 03 (2014) 017 [arXiv: 1311.4156] [INSPIRE].

[20] H. Gomez and E.Y. Yuan, N-point tree-level scattering amplitude in the new Berkovits" string, JHEP 04 (2014) 046 [arXiv: 1312.5485] [INSPIRE].

[21] T. Adamo, E. Casali and D. Skinner, Ambitwistor strings and the scattering equations at one loop, JHEP 04 (2014) 104 [arXiv: 1312.3828] [INSPIRE].

[22] Y. Geyer, A.E. Lipstein and L.J. Mason, Ambitwistor Strings in Four Dimensions, Phys. Rev. Lett. 113 (2014) 081602 [arXiv: 1404.6219] [INSPIRE].

[23] Y. Geyer, A.E. Lipstein and L. Mason, Ambitwistor strings at null infinity and (subleading) soft limits, Class. Quant. Grav. 32 (2015) 055003 [arXiv:1406.1462] [INSPIRE].

[24] E. Casali and P. Tourkine, Infrared behaviour of the one-loop scattering equations and supergravity integrands, JHEP 04 (2015) 013 [arXiv:1412.3787] [INSPIRE].

[25] T. Adamo and E. Casali, Scattering equations, supergravity integrands and pure spinors, JHEP 05 (2015) 120 [arXiv:1502.06826] [INSPIRE].

[26] E. Casali, Y. Geyer, L. Mason, R. Monteiro and K.A. Roehrig, New Ambitwistor String Theories, JHEP 11 (2015) 038 [arXiv:1506.08771] [InSPIRE].

[27] K. Ohmori, Worldsheet Geometries of Ambitwistor String, JHEP 06 (2015) 075 [arXiv: 1504.02675] [INSPIRE].

[28] Y. Geyer, L. Mason, R. Monteiro and P. Tourkine, Loop Integrands for Scattering Amplitudes from the Riemann Sphere, Phys. Rev. Lett. 115 (2015) 121603 [arXiv:1507.00321] [INSPIRE].

[29] N.E.J. Bjerrum-Bohr, P.H. Damgaard, P. Tourkine and P. Vanhove, Scattering Equations and String Theory Amplitudes, Phys. Rev. D 90 (2014) 106002 [arXiv:1403.4553] [INSPIRE].

[30] C. Baadsgaard, N.E.J. Bjerrum-Bohr, J.L. Bourjaily and P.H. Damgaard, Integration Rules for Scattering Equations, JHEP 09 (2015) 129 [arXiv: 1506.06137] [INSPIRE].

[31] C. Baadsgaard, N.E.J. Bjerrum-Bohr, J.L. Bourjaily and P.H. Damgaard, Scattering Equations and Feynman Diagrams, JHEP 09 (2015) 136 [arXiv:1507.00997] [INSPIRE].

[32] C. Baadsgaard, N.E.J. Bjerrum-Bohr, J.L. Bourjaily, P.H. Damgaard and B. Feng, Integration Rules for Loop Scattering Equations, JHEP 11 (2015) 080 [arXiv:1508.03627] [INSPIRE].

[33] S. He and E.Y. Yuan, One-loop Scattering Equations and Amplitudes from Forward Limit, Phys. Rev. D 92 (2015) 105004 [arXiv: 1508.06027] [INSPIRE].

[34] C. Baadsgaard, N.E.J. Bjerrum-Bohr, J.L. Bourjaily, S. Caron-Huot, P.H. Damgaard and B. Feng, New Representations of the Perturbative S-matrix, arXiv: 1509.02169 [INSPIRE].

[35] C. Kalousios, Scattering equations, generating functions and all massless five point tree amplitudes, JHEP 05 (2015) 054 [arXiv: 1502.07711] [INSPIRE].

[36] F. Cachazo and H. Gomez, Computation of Contour Integrals on $\mathcal{M}_{0, n}$, arXiv: 1505.03571 [INSPIRE].

[37] L. Dolan and P. Goddard, The Polynomial Form of the Scattering Equations, JHEP 07 (2014) 029 [arXiv:1402.7374] [INSPIRE]. 
[38] Y.-H. He, C. Matti and C. Sun, The Scattering Variety, JHEP 10 (2014) 135 [arXiv: 1403.6833] [INSPIRE].

[39] L. Stickelberger, Ueber eine Verallgemeinerung der Kreistheilung, Math. Ann. 37 (1890) 321.

[40] B. Sturmfels, Solving Systems of Polynomial Equations, Cbms Regional Conference Series in Mathematics, American Mathematical Society, Providenca U.S.A. (2002). Available online at https://math.berkeley.edu/ bernd/cbms.pdf.

[41] P. Parrilo, Algebraic Techniques and Semidefinite Optimization, Massachusetts Institute of Technology: MIT OpenCourseWare, Massachusetts Institute of Technology, Cambridge U.S.A. (2006). See lecture 15.

[42] D.R. Grayson and M.E. Stillman, Macaulay2, a software system for research in algebraic geometry. Available at http://www.math.uiuc.edu/Macaulay2/.

[43] W. Decker, G.-M. Greuel, G. Pfister and H. Schonemann, Singular 4-0-2 - A computer algebra system for polynomial computations, (2015). Available at http://www.singular.uni-kl.de.

[44] J. Gray, Y.-H. He, A. Ilderton and A. Lukas, STRINGVACUA: A Mathematica Package for Studying Vacuum Configurations in String Phenomenology, Comput. Phys. Commun. 180 (2009) 107 [arXiv:0801.1508] [INSPIRE].

[45] Z. Bern, J.J.M. Carrasco and H. Johansson, New Relations for Gauge-Theory Amplitudes, Phys. Rev. D 78 (2008) 085011 [arXiv: 0805.3993] [INSPIRE].

[46] H. Kawai, D.C. Lewellen and S.H.H. Tye, A Relation Between Tree Amplitudes of Closed and Open Strings, Nucl. Phys. B 269 (1986) 1 [inSPIRE].

[47] Z. Bern, L.J. Dixon, M. Perelstein and J.S. Rozowsky, Multileg one loop gravity amplitudes from gauge theory, Nucl. Phys. B 546 (1999) 423 [hep-th/9811140] [INSPIRE].

[48] N.E.J. Bjerrum-Bohr, P.H. Damgaard, B. Feng and T. Sondergaard, Gravity and Yang-Mills Amplitude Relations, Phys. Rev. D 82 (2010) 107702 [arXiv:1005.4367] [InSPIRE].

[49] N.E.J. Bjerrum-Bohr, P.H. Damgaard, B. Feng and T. Sondergaard, New Identities among Gauge Theory Amplitudes, Phys. Lett. B 691 (2010) 268 [arXiv:1006.3214] [INSPIRE].

[50] N.E.J. Bjerrum-Bohr, P.H. Damgaard, B. Feng and T. Sondergaard, Proof of Gravity and Yang-Mills Amplitude Relations, JHEP 09 (2010) 067 [arXiv: 1007.3111] [INSPIRE].

[51] S.J. Parke and T.R. Taylor, An Amplitude for n Gluon Scattering, Phys. Rev. Lett. 56 (1986) 2459 [INSPIRE].

[52] F.A. Berends and W.T. Giele, Recursive Calculations for Processes with n Gluons, Nucl. Phys. B 306 (1988) 759 [INSPIRE].

[53] B. Feng, J. Rao and K. Zhou, On Multi-step BCFW Recursion Relations, JHEP 07 (2015) 058 [arXiv: 1504.06306] [INSPIRE].

[54] D.A. Kosower, Next-to-maximal helicity violating amplitudes in gauge theory, Phys. Rev. D 71 (2005) 045007 [hep-th/0406175] [INSPIRE].

[55] M.-x. Luo and C.-k. Wen, Recursion relations for tree amplitudes in super gauge theories, JHEP 03 (2005) 004 [hep-th/0501121] [INSPIRE]. 